\title{
HEEGAARD SPLITTINGS OF BRANCHED COVERINGS OF $S^{3}$
}

\author{
BY \\ JOAN S. BIRMAN( $\left.{ }^{1}\right)$ AND HUGH M. HILDEN( ${ }^{2}$ )
}

ABSTRACT. This paper concerns itself with the relationship between two seemingly different methods for representing a closed, orientable 3-manifold: on the one hand as a Heegaard splitting, and on the other hand as a branched covering of the 3-sphere. The ability to pass back and forth between these two representations will be applied in several different ways:

1. It will be established that there is an effective algorithm to decide whether a 3-manifold of Heegaard genus 2 is a 3-sphere.

2. We will show that the natural map from 6-plat representations of knots and links to genus 2 closed oriented 3-manifolds is injective and surjective. This relates the question of whether or not Heegaard splittings of closed, oriented 3manifolds are "unique" to the question of whether plat representations of knots and links are "unique".

3. We will give a counterexample to a conjecture (unpublished) of W. Haken, which would have implied that $\boldsymbol{S}^{\mathbf{3}}$ could be identified (in the class of all simply-connected 3-manifolds) by the property that certain canonical presentations for $\pi_{1} s^{3}$ are always "nice".

The final section of the paper studies a special class of genus 2 Heegaard splittings: the 2-fold covers of $S^{3}$ which are branched over closed 3-braids. It is established that no counterexamples to the "genus 2 Poincaré conjecture" occur in this class of 3-manifolds.

1. Introduction. We begin our study in $\$ 2$ with a brief discussion of several different methods for presenting links, and the relationship between them. In particular, we discuss two ways to form a link from a braid, as a "closed $n$ braid", or as a "plat". These results will be used later. $\$ 3$ contains a brief review of known facts about twist maps on surfaces.

In $\$ 4$ we develop the central results of this paper. We study the relationship between representations of closed, orientable 3-manifolds by Heegaard splittings, and as branched coverings of $S^{3}$. Theorem 1 is a generalization of a theo-

Received by the editors July 23, 1973 and, in revised form, October 7, 1974.

AMS (MOS) subject classifications (1970). Primary 55A10, 55A25, 55A35, 55A40, 55A05, 02E10; Secondary 20F05, 20 F55.

Key words and phrases. Poincaré conjecture, Heegaard splittings, branched covering spaces, three-manifolds, three-sphere, Smith conjecture, plats, links, closed braids, bridge number.

(1) The work of the first author was supported in part by NSF Grant \#GP-38479.

(2) The work of the second author was supported in part by NSF Grant \#GP-34059. 
rem due to Alexander [1], which states that every closed, orientable 3-manifold may be represented as a branched covering of $S^{\mathbf{3}}$, branched over a link. Our method of proof is very different from Alexander's, and moreover it contains certain extra information about the relationship between Heegaard genus and Heegaard "sewing maps", on the one hand, and the branch set and number of sheets, on the other hand. In the general case, the covering will not be regular, however in certain special cases (notably all genus 2 Heegaard splittings) the covering will not only be regular, but even cyclic.

The idea used in the proof of Theorem 1 may be specialized to the case of Heegaard splittings which enjoy a certain symmetry. We give these 3-manifolds the name " $p$-symmetric Heegaard splittings", and establish (in Theorems 2-5) the relationship between $p$-symmetric Heegaard splittings and $p$-fold cyclic coverings of $S^{3}$. The results in Theorems 2-5 were announced by the authors in [8]. We note that Theorem 5 was also discovered independently by 0 . Ja. Viro [32]. Also, that Theorem 5 generalizes a result due to Schubert [30].

$\S \S 5-7$ are concerned with applications of the results of $\S 4$. In $\S 5$ we give an algorithm to decide whether a 2-symmetric 3-manifold is $S^{\mathbf{3}}$. Our algorithm applies to all 3-manifolds of Heegaard genus $g \leqslant 2$, and to a proper subset of 3 manifolds of Heegaard genus $g \geqslant 3$. The algorithm was announced by the authors in [8]. At the conclusion of $\S 5$ the algorithm is applied to give a new proof that every genus 1 homology sphere is $S^{3}$.

In $\S 6$ we define a function $F$ from equivalence classes of plat presentations of knots and links to equivalence classes of Heegaard splittings of closed, oriented 3-manifolds and we show that $F$ is injective and surjective.

$\$ 7$ contains applications to problems concerning presentations for $\pi_{1} S^{3}$. In Lemma 13 we give an algorithm for obtaining a "canonical" presentation for the fundamental group of a 3-manifold which is defined by a Heegaard splitting, where it is assumed that the action of the "sewing maps" on the fundamental group of the Heegaard surface is known. In Lemma 14 we characterize the class of sewing maps which have the property that the corresponding Heegaard splittings define $S^{\mathbf{3}}$. These two lemmas are used in Theorem 8 to obtain a counterexample to a conjecture of $W$. Haken about canonical presentations of $\pi_{1} S^{3}$-an unfortunate result, because it points up the difficulty in solving the "trivial group problem" for 3-manifolds. Finally, at the end of $\$ 7$, we discuss symmetries in our canonical presentations for fundamental groups of "2-symmetric" 3-manifolds (Theorem 9).

The final section of the paper contains a brief proof that one will not find counterexamples to the Poincare conjecture among 2-fold coverings of $S^{3}$ which are branched over closed 3-braids. This may be regarded as a small step in an attempt to prove the "genus 2 Poincaré conjecture". 
ACKnowledgement. The authors wish to thank Professor Jose M. Montesinos, Universidad Complutense, Madrid, Spain, who pointed out an error in an earlier version of Theorems 7 and 8, and who also helped to close a gap in our proof of Theorem 11.

2. Links, closed braids and plats. A link will be understood to mean the union of $\mu \geqslant 1$ tame polygonal disjoint simple closed curves in $S^{3}$. The term $k$ not will be used when we wish to stress the fact that $\mu=1$. Two links are equivalent if there is an orientation-preserving homeomorphism of $S^{3} \rightarrow S^{3}$ which maps one onto the other. A link type is the equivalence class of a tame link.

Let $E^{2}$ be the Euclidean plane, let $\Pi_{n} E^{2}$ be the $n$-fold product space, and let $F_{n} E^{2}$ denote the subspace of $\Pi_{n} E^{2}$ consisting of all points $\left(p_{1}, \ldots, p_{n}\right) \in$ $\Pi_{n} E^{2}$ which have the property $p_{i} \neq p_{j}$ if $i \neq j$. Let $B_{n} E^{2}$ denote the quotient space of $F_{n} E^{2}$ obtained by identifying points which differ only by a permutation. Let $\left(p_{10}, \ldots, p_{n 0}\right) \in F_{n} E^{2}$, and let $\vec{p}_{0}$ be its image in $B_{n} E^{2}$. The classical braid group $B_{n}$ is defined to be in the fundamental group $\pi_{1}\left(B_{n} E^{2}, \vec{p}_{0}\right)$. Each element $\beta \in B_{n}$ may be represented by a loop $(I, \partial I) \rightarrow\left(B_{n} E^{2}, \vec{p}_{0}\right)$ which lifts to a path $(I, 0) \rightarrow\left(F_{n} E^{2},\left(p_{10}, \ldots, p_{n 0}\right)\right)$. This path may be described by the image set $\left(p_{1}(t), \ldots, p_{n}(t)\right), 0 \leqslant t \leqslant 1$, where $\left(p_{1}(0), \ldots, p_{n}(0)\right)=\left(p_{10}\right.$, $\left.\ldots, p_{n 0}\right)$ and $\left(p_{1}(1), \ldots, p_{n}(1)\right)=\left(p_{\mu_{1} 0}, \ldots, p_{\mu_{n} 0}\right)$ is a permutation of $\left(p_{10}, \ldots, p_{n 0}\right)$. We will call this image set, or any other obtained in a similar manner from another representative of the same element $\beta \in B_{n}$, a geometric $n$ braid. We may think of a geometric $n$-braid as a configuration of $n$ "strings" suspended between parallel planes $t=0$ and $t=1$ in $E^{3}$.

The braid group $B_{n}$ has been studied extensively in the literature. For a review of the literature on braids and related topics, see [6]. Original references include [3], [4] , [9] , [14], [19]. We will make particular use of the following well-known facts:

1. The braid group may be generated by a standard set of "elementary" braids $\sigma_{1}, \ldots, \sigma_{n-1}$, where $\sigma_{i}$ denotes a braid in which the $i$ th string crosses over the $(i+1)$ st, as illustrated in Figure 1 .

2. The group $B_{n}$ has a faithful representation as the full group of autohomeomorphisms of an $n$-punctured disc, where admissible maps are required to keep the boundary fixed pointwise. The elementary braid $\sigma_{i}$ may be visualized in this representation as a twist which interchanges the $i$ th puncture and the $(i+1)$ st puncture, and is the identity map outside a disc which encloses these two punctures, but avoids all other punctures. Generators $x_{1}, \ldots, x_{n}$ can be chosen for the fundamental group of the punctured disc in such a way that the elementary braid $\sigma_{i}$ induces the automorphism $\sigma_{i}\left(x_{i}\right)=x_{i+1}, \sigma_{i}\left(x_{i+1}\right)=$ $x_{i+1}^{-1} x_{i} x_{i+1}, \sigma_{i}\left(x_{j}\right)=x_{j}$ is $j \neq i, i+1$. 


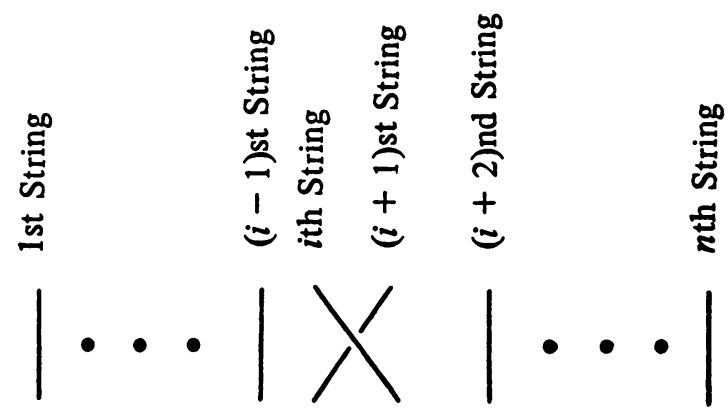

FIgURE 1. Geometric $n$-braid representing the element $\sigma_{i}$

A link type is said to be represented as a closed n-braid if it has a representative which is obtained from a geometric $n$-braid by identifying the points $p_{i}(0)$ and $p_{i}(1)$ for each $i=1, \ldots, n$. It was proved by Alexander [2], [6] that every link type may be represented by a closed $n$-braid for some (nonunique) integer $\boldsymbol{n}$. The braid number of a link is the smallest such integer.

A link type is said to be displayed as a $2 m$-plat if it is obtained from a geometric braid on $2 m$ strings by identifying the points $p_{2 i-1}(0)$ and $p_{2 i}(0)$, and also $p_{2 i-1}(1)$ and $p_{2 i}(1)$, for each $i=1, \ldots, m$. It was established by Reidemeister in [29] that every link type may be represented as a $2 m$-plat for some (nonunique) integer $m$. For another proof, see [6].

A link type is said to have an $m$-bridge presentation if it is represented as the union of two subsets $A, B$ of $E^{3}$, parametrized by $x, y, z$ coordinates, as follows:

$A$ is the disjoint union of $m$ arcs lying in the plane $z=0$, with endpoints in the set $\{(i, 0,0) ; i=1, \ldots, 2 m\}$.

$B$ is the disjoint union of $m$ arcs lying in the plane $y=0$, the $j$ th arc having its endpoints at $(2 j-1,0,0)$ and $(2 j, 0,0)$, and its interior in the subset of the plane defined by the condition $z>0$.

The relationship between plat and bridge representations of a link, and between bridge number and braid number, is expressed by:

LEMMA 1. If a link type is represented in an m-bridge presentation, then it may also be represented as a $2 \mathrm{~m}$-plat; conversely, if it is represented as a $2 \mathrm{~m}$ plat, then it may also be represented in an m-bridge presentation. Thus the bridge number is also the smallest integer $b$ such that the link can be represented by a $2 b$-plat.

Proof. See [6].

LEMMA 2. The bridge number of a link is less than or equal to its braid number. 
Proof. See [6].

3. Twist maps on surfaces. Let $Y_{g}^{*}$ be a solid handlebody of genus $g$, and let $\partial Y_{g}$ denote its surface (a closed, orientable surface of genus $g$ ). In the sections which follow we will need to know certain information about self-homeomorphisms of $\partial Y_{g}$.

Let $c$ be a simple closed curve on the surface $\partial Y_{g}$. Let $N_{c}$ be a neighborhood of $c$ which is homeomorphic to a cylinder. Assume that $N_{c}$ has cylindrical coordinates $(y, \theta),-1 \leqslant y \leqslant+1,0 \leqslant \theta \leqslant 2 \pi$. A twist $t_{c}$ about $c$ is defined by the identity map outside $N_{c}$, with $t_{c}(y, \theta)=(y, \theta+\pi(y+1))$.

It was proved by M. Dehn [11] that every homeomorphism of $\partial Y_{g}$ is isotopic to a product of twists. Dehn's result was later reproved and simplified by W.B.R. Lickorish [17], [18], who showed:

LEMMA 3 (LICKORISH [18]). Let $\Phi: \partial Y_{g} \rightarrow \partial Y_{g}$ be a homeomorphism. Then $\Phi$ is isotopic to a product of the twists $t_{c_{1}}, \ldots, t_{c_{3 g-1}}$ about the curves $c_{1}, \ldots, c_{3 g-1}$ illustrated in Figure 2.
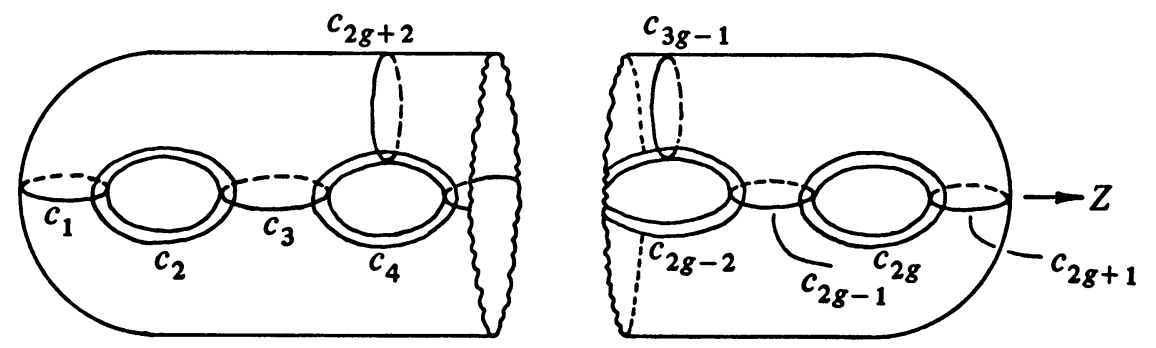

FIGURE 2. Generators for the mapping class group of a closed, orientable surface of genus $g$

4. Heegaard splittings of branched coverings of $S^{3}$. Let $M, N$ be triangulated $n$-dimensional manifolds, and let $\pi: M \rightarrow N$ be a simplicial map. The map $\pi$ is said to be a branched covering space projection if the restriction of $\pi$ to the complement of the $(n-2)$-dimensional skeleton of the triangulation is a covering space projection. The branch set $A \subset N$ is the set of points $z \in N$ which have the property that $z$ has no neighborhood $U$ such that the restriction of $\pi$ to an arc-component of $\pi^{-1}(U)$ is a covering. The set $L=\pi^{-1}(A)$ will be referred to as the branch cover, and we will say that $M$ is a covering space of $N$, branched over $A$. We will also refer to the pair $(M, L)$ as a branched covering of $(N, A)$, writing $\pi:(M, L) \rightarrow(N, A)$.

Let $(M . L)$ be a branched covering of $(N, A)$, with projection $\pi$. Let $\hat{\pi}$ be the restriction of $\pi$ to $(M-L)$. Then $\hat{\pi}:(M-L) \rightarrow(N-A)$ is an ordinary covering space projection, which will be referred to as the associated unbranched covering space. 
We will say that $M$ is a $p$-fold cyclic covering of $N$ branched over $A$ if the group $\hat{\pi}_{*} \pi_{1}(M-L)$ is the kernel of a homeomorphism from $\pi_{1}(N-A)$ onto a cyclic group of order $p$.

It was proved by Alexander in [1] that every closed, orientable 3-manifold may be represented as a branched covering of $S^{3}$. Alexander also stated (without proof) that the branch set may be chosen to be a 1-manifold. This fact has been widely accepted in the literature, although no published proof exists. (The gap will, however, be filled in Theorem 1 , below. $\left({ }^{3}\right)$

We will also be interested in a second method of representing closed, orientable 3-manifolds, as Heegaard splittings. Let $Y_{\boldsymbol{g}}$ be a solid handlebody of genus $g$. Let $Y_{g}^{\prime}$ be a second copy of $Y_{g}$, and let $\tau: Y_{g} \rightarrow Y_{g}^{\prime}$ be the map that identifies a point $z \in Y_{g}$ with its corresponding point $z^{\prime} \in Y_{g}^{\prime}$. Let $\Phi$ be an orientationpreserving self-homeomorphism of $\partial Y_{g}$. Then we may use $\Phi$ to define a map which "sews" $\partial Y_{g}$ to $\partial Y_{g}^{\prime}$ by the rule

$$
\tau \Phi(z)=z, \quad z \in \partial Y_{g} .
$$

The identification space $Y_{g} \cup_{\tau \Phi} Y_{g}^{\prime}$ will be a closed, orientable 3-manifold which is represented by a Heegaard splitting of genus $g$. It is a classical result that every closed, orientable 3-manifold may be obtained in this way, for some (nonunique) integer $g$ and surface homeomorphism $\Phi$. A 3-manifold which is so represented will be said to have Heegaard genus $g$ if it admits a Heegaard splitting of genus $g$, but no Heegaard splitting of genus $<g$.

The following result will be established below:

THEOREM 1.* Let $M=Y_{g} \cup_{\tau \Phi} Y_{g}^{\prime}$ be a closed orientable 3-manifold of Heegaard genus $g$.

(1.1) If $g \leqslant 2$, then $M$ may be represented as a 2 -fold cyclic covering of $S^{3}$, branched over a link of bridge number $g+1$.

(1.2) If $g \geqslant 3$, then $M$ may be represented as a $(4 g-4)$-sheeted branched covering of $S^{3}$, with the branching set a 1-manifold of at most $4 g-4$ components.

Theorem 1 generalizes a result of Alexander [1]. The method used here to prove Theorem 1 is very different from that employed by Alexander, and may be of some interest in its own right. Statement (1.1) of Theorem 1 was established by the authors in [8], and will also be reproved below in a somewhat more general context. We remark that our proof of Theorem 1 is constructive in that it

(3) See W. B. R. Lickorish, 3-manifolds as branched covers, Proc. Cambridge Philos. Soc. 74 (1973), 449-451, for another new proof of Alexander's theorem, different from that given here.

*Note added in proof. Hilden and Montesinos have shown that any closed oriented 3-manifold is a 3-fold branched covering of $S^{3}$. See Bull. Amer. Math. Soc. 80 (1974), pp. 845-846 and 1243-1244. 
allows us to relate the branching set in an explicit manner to the Heegaard "sewing map".

We will also be interested in certain special types of Heegaard splittings, which we will refer to as $p$-symmetric Heegaard splittings. Let $Y_{g}, Y_{g}^{\prime}, \tau, \Phi$ be as defined above. It will be assumed further that there is given a piecewise-linear homeomorphism $P . E^{3} \rightarrow E^{3}$ of period $p$, and that $Y_{g}$ is left invariant under the action of $P$. Note that the homeomorphisms $P$ and $\tau$ define in a natural way a transformation $P^{\prime}=\tau P_{\tau^{-1}}$ which acts on $Y_{g}^{\prime}$, that $P^{\prime}$ also has period $p$, and that $Y_{g}^{\prime}$ is left invariant under the action of $P^{\prime}$. The Heegaard splitting $Y_{g} \cup_{\tau \Phi} Y_{g}^{\prime}$ will be said to be p-symmetric if

(i) There is an integer $p_{0}$, with $1 \leqslant p_{0} \leqslant p$, such that

$$
(\Phi)\left(P \mid \partial Y_{g}\right)\left(\Phi^{-1}\right)=\left(P \mid \partial Y_{g}\right)^{p_{0}} \text {. }
$$

(ii) The orbit space of $Y_{g}$ under the action of $P$ is a 3-ball.

(iii) The fixed point set of $P=$ fixed point set of $P^{k}$ for each $k, 1 \leqslant k<p$.

(iv) The image of the fixed point set of $P$ is an unknotted set of arcs in the ball $Y_{g} / P$.

It will be proved below that the class of 3-manifolds which admit p-symmetric Heegaard splittings coincides with the class of 3-manifolds which may be represented as $p$-fold cyclic coverings of $S^{\mathbf{3}}$ branched over a link.

To state the latter result precisely, we define a new concept: the p-symmetric Heegaard genus of a 3-manifold $M$ is the smallest integer $g$ such that $M$ admits a $p$-symmetric Heegaard splitting of genus $g$. We will establish

THEOREM 2. Let $g \geqslant 0, p \geqslant 1, b \geqslant 1$ be integers which are related by the equation

$$
g=(b-1)(p-1)
$$

Then every closed, orientable 3-manifold of p-symmetric Heegaard genus $g$ admits a representation as a p-fold cyclic covering of $S^{3}$ branched over a link $\left({ }^{4}\right)$ of bridge number $\leqslant b$.

THEOREM 3. The p-fold cyclic covering of $S^{3}$ branched over a knot of braid number $b$ is a closed, orientable 3-manifold of p-symmetric Heegaard genus $g \leqslant(b-1)(p-1)$.

THEOREM 4. Every closed, orientable 3-manifold of 2-symmetric Heegaard genus $g$ is a 2-fold covering of $S^{3}$ branched over a $(g+1)$-bridge link.

THEOREM 5. Every closed, orientable 3-manifold of Heegaard genus $g \leqslant 2$

(4) The reader is reminded that a "link" may have $\mu \geqslant 1$ components, while a "knot" is a link of 1 component. 
is a 2-fold covering of $S^{3}$ branched over a link of bridge number $g+1$. Converse$l y$, the 2-fold covering of $S^{3}$ branched over a link of bridge number $b \leqslant 3$ is a closed, orientable 3-manifold of Heegaard genus $b-1$.

Theorems 2-5 were announced in [8]. Theorem 5 was also discovered independently by 0 . Ja. Viro in [32]. We note that Theorem 5 reduces to a result of Schubert [30] in the case $g=1$ and $b=2$; our method of proof is, however, different from his method.

We will begin our proof by establishing Theorems 2 and 3. Theorems 4 and 5 will then follow, easily. Statement (1.1) of Theorem 1 is implied by Theorem 5. The proof of statement (1.2) of Theorem 1 is somewhat more complicated, and will be treated last.

Note that all of the results above are trivially true if $g=0$, hence we will always assume $g \geqslant 1$.

Proof of Theorem 2. The definition of a P-symmetric Heegaard splitting implies that the map $q: Y_{g} \rightarrow Y_{g} / P$ is a branched covering space. The restriction of $q$ to $\partial Y_{g}$ defines a branched covering space $\partial Y_{g} \rightarrow \partial\left(Y_{g} / P\right)$, and since $Y_{g} / P$ is a 3-ball, it follows that its boundary is a 2-sphere. Since the covering is cyclic of order $p$, standard results on classification of surfaces imply that $g, p$ and $b$ are related by (3).

To elucidate the structure of the branch set, it will be convenient to use an explicit representation for a handlebody $Y_{g}$ of genus $g$ as a subset of Euclidean 3-space $E^{3}$. This handlebody will be constructed in such a way that it invariant under a rotation $P$ of period $p$ about the $z$-axis of $E^{3}$, and also in such a way that $Y_{g} / P$ is a 3-ball. The handlebody $Y_{g}$ will then be used to construct a $p$ symmetric 3-manifold $M=Y_{g} \cup_{\tau \Phi} Y_{g}^{\prime}$.

Let $(r, \theta, z)$ denote cylindrical coordinates in $E^{3}$. Let $g \geqslant 1, p \geqslant 2, b \geqslant 2$ be integers which satisfy the equality (3). Let $Y_{g}=Y_{b, p}$ denote a handlebody of genus $g=(b-1)(p-1)$, which will now be described in terms of its cylindrical coordinates.

Let $K(b, p)$ be the union of the set of line segements $\{[(1,2 \pi j / p, i)$, $(0,0, i)] ; j=1, \ldots, p ; i=1, \ldots, b\}$ and $\{[(1,2 \pi j / p, 1),(1,2 \pi j / p, b)]$, $j=1, \ldots, p\}$. (See Figure 3.) The handlebody $Y_{b, p}$ will be regarded as the set of points whose distance from $K(b, p)$ is less than or equal to $(1 / 10)^{p}$. Let $P$ denote rotation about the $z$ axis by $2 \pi / p$. It is easily verified that $K(b, p)$ and $Y(b, p)$ are left invariant as sets by $P$, that $Y(b, p)$ has genus $(b-1)(p-1)$ and that $F_{b, p}=\left\{(r, \theta, z) \in Y_{b, p} \mid \pi / p \leqslant \theta \leqslant 3 \pi / p\right\}$ is a fundamental set for $P$. (See Figure 4.)

The natural projection $Y_{b, p} \rightarrow Y_{b, p} / P$ is a branched covering space projection. The branch cover (preimage of the branch set), denoted by $L_{b, p}$, is the point set $Y_{b, p} \cap z$ axis. It consists of the $b$ disjoint arcs $\left[\left(0,0, i-1 / 10^{p}\right)\right.$, 


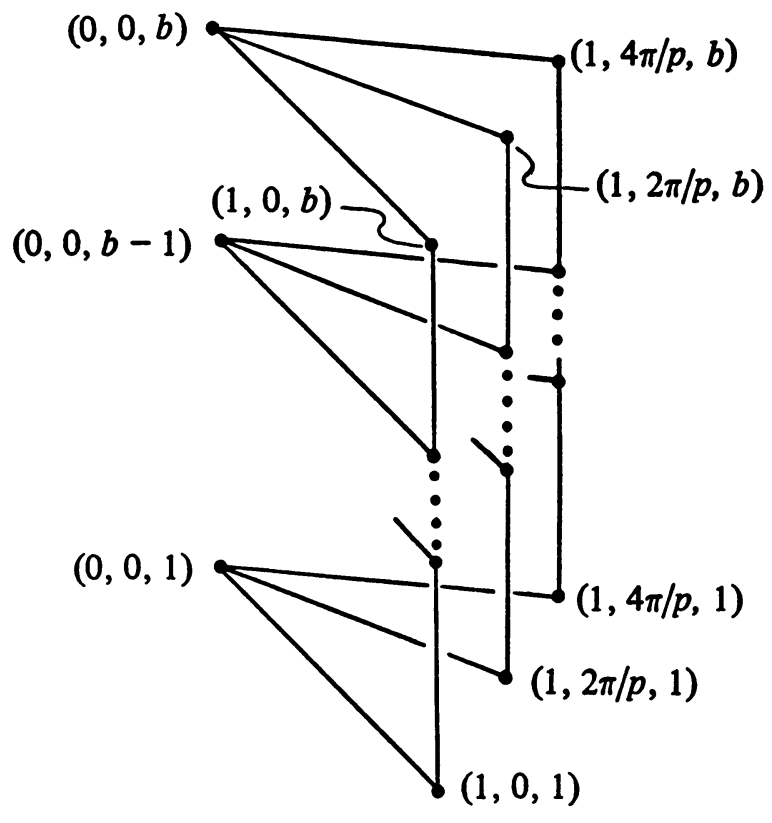

Figure 3. Part of the set $K(b, p)$

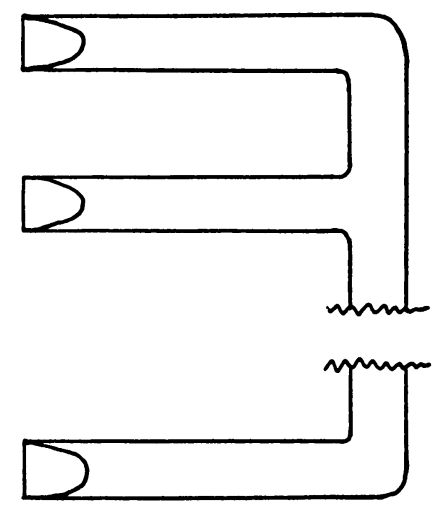

Figure 4. Fundamental set $F_{b, p}$ for action of $P$ on $K(b, p)$

$\left.\left(0,0, i+1 / 10^{p}\right)\right]$ where $i=1, \ldots, b$. The underlying space $\left(Y_{b, p} / P, L_{b, p} / P\right)$ is obtained by identifying the points $(r, \pi / p, z)$ and $(r, 3 \pi / p, z)$ in $F_{b, p}$. It is a 3-ball with $b$ distinguished unknotted, unlinked arcs. For simplicity we will hereafter denote $\left(Y_{b, p} / P, L_{b, p} / P\right)$ by $\left(D, A_{b}\right)$.

Let $Y_{b, p}^{\prime}$ be another copy of $Y_{b, p}$, and let $\tau: Y_{b, p} \rightarrow Y_{b, p}^{\prime}$ be the map that identifies a point $z \in Y_{b, p}$ with its corresponding point $z^{\prime} \in Y_{b, p}^{\prime}$. For simplicity, we will use the same symbol $\tau$ to denote the map that identifies a 
point $z$ with its corresponding point $z^{\prime}$ whether the domain be $Y_{b, p}, \partial Y_{b, p}$, $L_{b, p}, D$ or $\partial D$. It will be clear from the context what we intend the domain to be. Recall that $Y_{b, p}$ is invariant under the rotation $P$ of $2 \pi / p$ about the $z$-axis. It then follows that $Y_{b, p}^{\prime}$ will be invariant under the map $P^{\prime}=\tau P \tau^{-1}$, which also has period $p$. In a similar fashion, we may use $\tau$ to define the branch cover $L_{b, p}^{\prime}=\tau L_{b, p}^{\prime} \tau^{-1}$, the set $\left(Y_{b, p}^{\prime} / P^{\prime}, L_{b, p}^{\prime} / P^{\prime}\right)=\left(D^{\prime}, A_{b}^{\prime}\right)$ and the branched covering space projection $\pi^{\prime}:\left(Y_{b, p}^{\prime}, L_{b, p}^{\prime}\right) \rightarrow\left(D^{\prime}, A_{b}^{\prime}\right)$, all in a natural way.

Let $\Phi$ be an orientation-preserving homeomorphism which satisfies the condition (2). Then $M=Y_{b, p} \cup_{\tau \Phi} Y_{b, p}^{\prime}$ is a closed orientable 3-manifold which is exhibited as a $p$-symmetric Heegaard splitting. Let $\varphi=\pi \Phi \pi^{-1}$. Then the map $\pi \cup \pi^{\prime}: Y_{b, p} \cup_{\tau \Phi} Y_{b, p}^{\prime} \rightarrow D \cup_{\tau \varphi} D^{\prime}$ is a $p$-fold cyclic covering space projection branched over $A_{b} \cup_{\tau \varphi} A_{b}^{\prime}$. Since $D \cup_{\tau \varphi} D^{\prime}$ is a Heegaard splitting of genus 0 , it can only be $S^{3}$. Hence $M=Y_{b, p} \cup_{\tau \Phi} Y_{b, p}^{\prime}$ is a $p$-fold cyclic covering space of $S^{3}$, branched over $A_{b} \cup_{\tau \varphi} A_{b}^{\prime}$.

It remains to show that $A_{b} \cup_{\tau \varphi} A_{b}^{\prime}$ is a link which is represented by a $2 b$ plat. To see this, recall that the point set $A_{b}$ is a collection of $b$ unknotted, unlinked $\operatorname{arcs} A_{b 1}, \ldots, A_{b b}$ which are contained in the 3-ball $D$, with $\partial A_{b}$ a set of $2 b$ points on $\partial D$ (cf. Figure 5). The point set $A_{b}^{\prime}$ is the image of $A_{b}$ under $\tau$.

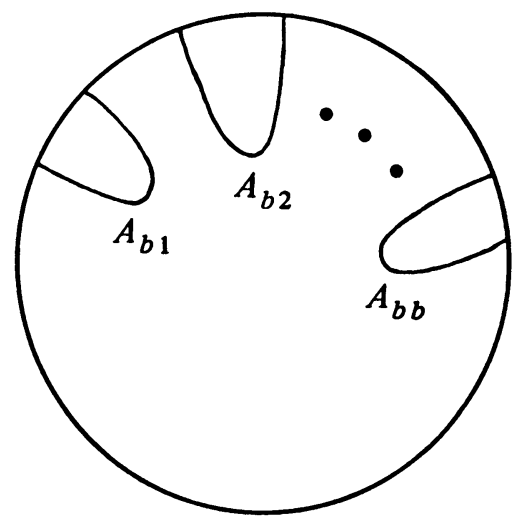

Figure 5. The set $\left(D, A_{b}\right)$

The sewing map $\tau \varphi=\tau \pi \Phi \pi^{-1}$ has the property $\tau \varphi\left(\partial A_{b}\right)=\partial A_{b}^{\prime}$. It is now clear that $A_{b} \cup_{\tau \varphi} A_{b}^{\prime}$ can only be a 1 -manifold which is represented as a $2 b$-plat imbedded in the 3-sphere $D \cup_{\tau \varphi} D^{\prime}$. This completes the proof of Theorem 2.

We turn our attention next to Theorem 3 , which is a partial converse to Theorem 2. As a preparatory step, we investigate further the branched covering space projection $\pi: Y_{b, p} \rightarrow D$ constructed in the proof of Theorem 2, considering in particular the restriction of this branched covering space projection to the surface $\partial Y_{b, p}$. The associated unbranched covering space will be the regular cyclic 
covering space $\left(\hat{\pi}, \partial Y_{b, p}-\partial L_{b, p}, \partial D-\partial A_{b}\right)$ of the 2-sphere $\partial D$ with the $2 b$ points $\partial A_{b}$ removed. We next describe a set of generators for the fundamental group $\pi_{1}\left(\partial D-\partial A_{k}\right)$ of the base space.

The point set

$$
\left(\partial F_{b, p} \cap \text { the half-plane } \theta=2 \pi / p\right) \cup\left\{(r, \theta, z) \in \partial F_{b, p} \mid \theta=\pi / p \text { or } 3 \pi / p\right\}
$$

describes a simple closed curve $c$ on $\partial D$ that contains all the branch points. Pick a point $x_{0}$ not on $c$. Let $S_{1}, Q_{1}^{\circ}, \ldots, S_{b}, Q_{b}$ be the ordered array of points on $\partial D$ whose coordinates on $F_{b, p}$ are $\left(0,0,1-1 / 10^{p}\right),\left(0,0,1+1 / 10^{p}\right), \ldots$, $\left(0,0, b+1 / 10^{p}\right)$. These are now the branch points. Let $s_{i}$ be a simple closed curve which starts at $x_{0}$, first intersects the curve $c$ between the points $S_{i}$ and $S_{i+1}$, and then intersects $c$ exactly one more time, enclosing the point $S_{i}$ (but no other point $S_{j}$ or $\left.Q_{k}\right)$. Let $q_{i}$ be a simple closed curve which starts at $x_{0}$, first intersects $c$ between $Q_{i-1}$ and $Q_{i}$ (where $Q_{0}=S_{b}$ ), and then intersects $c$ exactly one more time, enclosing the point $Q_{i}$ (but no other point $Q_{j}$ or $S_{k}$ ). Notice that $s_{i}$ and $q_{i}$ have opposite orientation. The group $\pi_{1}\left(\partial D-\partial A_{k}\right)$ is a free group, which admits the presentation

$$
\left\langle s_{1}, \ldots, s_{b}, q_{1}, \ldots, q_{b} ; s_{1} q_{1}^{-1} s_{2} \cdots q_{b}^{-1}\right\rangle .
$$

The subgroup belonging to the covering space $\pi:\left(\partial Y_{b, p}-\partial L_{b, p}\right) \rightarrow\left(\partial D-\partial A_{b}\right)$ is the set of words which have total exponent sum a multiple of $p$.

LEMMA 4. If $p=2$, every orientation-preserving homeomorphism of $\partial D$ which leaves the set $\partial D \cap A_{b}$ invariant lifts to a homeomorphism of $\partial Y_{b, p}$ which leaves the set $\partial Y_{b, p} \cap L_{b, p}$ invariant. If $p \geqslant 3$, an orientation-preserving homeomorphism of $\partial D$ which leaves the set $\partial D \cap A_{b}$ invariant lifts to a homeomorphism of $\partial Y_{b, p}$ which leaves the set $\partial Y_{b, p} \cap L_{b, p}$ invariant if and only if the homeomorphism leaves the point set $S=\left\{S_{1}, \ldots, S_{b}\right\}$ invariant.

Proof of Lemma 4. It is well known that a homeomorphism $\eta$ of $\partial D$ which fixes the branch points as a set lifts if and only if $\eta_{*}$ leaves the subgroup of the covering invariant [22]. If $\eta$ preserves orientation, then $\eta_{*}$ necessarily takes each $s$-type generator (respectively $q$-type generator) into a conjugate of another $s$-generator (respectively $q$-generator) or into a conjugate of some $q^{-1}$ (respectively $s^{-1}$ ). (To see this, think of $s_{i}$ as a curve which traverses an arc $\alpha$ from the base point to a point near $S_{i}$, then winds about $S_{i}$ in a small circular path, and then traverses $\alpha$ in the reverse direction, and recall also that $s_{i}$ and $q_{i}$ wind in opposite directions.) If $p=2$, the elements $s_{i}$ and $w q_{j}^{-1} w^{-1}$ have the same exponent sum $(\bmod 2)$, so that every $\eta_{*}$ leaves the subgroup of the covering invariant. But if $p>2$, then $s_{i}$ and $w q_{j}^{-1} w^{-1}$ do not have the same exponent sum $(\bmod b)$ and the subgroup of the covering is left invariant if and only if $\eta_{*}$ 
maps s-generators into conjugates of other s-generators. This happens if and only if $\eta_{*}$ leaves the set $S=\left\{S_{1}, \ldots, S_{b}\right\}$ invariant. This proves Lemma 4 .

One more preparatory lemma is needed for the proof of Theorem 3. Ob. serve that the statement of Theorem 3 refers only to knots, not to links. This is not surprising, in view of the following:

LEMMA 5. There is a unique p-fold cyclic covering of $S^{3}$ branched over a link of $\mu \geqslant 1$ components if and only if either $p=2$ or $\mu=1$.

Proof of Lemma 5. The $p$-fold coverings of $S^{3}$ branched over a link $L$ are in 1-1 correspondence with nontrivial homomorphisms $w$ of $\pi_{1}\left(S^{3}-L\right)$ into the symmetric group on $p$ letters, by the following rule: one considers $p$ copies of $S^{3}$, each with the distinguished subset $L$, and identifies them along $L$ according to pasting instructions given by $w$. If the covering is cyclic, then the correspondence will be with equivalence classes of nontrivial homomorphisms of $\pi_{1}\left(S^{3}-L\right)$ onto the cyclic group $Z_{p}$, where two such homomorphisms $\alpha, \beta$ are equivalent if there is an automorphism $\delta$ of $Z_{p}$ such that $\alpha=\beta \delta$. Since $Z_{p}$ is abelian, any such homomorphism factors through the commutator quotient group of $\pi_{1}\left(S^{3}-L\right)$, which is a $\mu$-fold direct sum $Z \oplus \cdots \oplus Z$. The pre-images of the generators of the summands are necessarily meridians of $L$, and there is one summand $Z$ for each component of the link. The meridians of the link are mapped onto the elements $(0, \ldots, 0,1,0, \ldots, 0)$, where the meridian of the $k$ th component has an entry 1 in the $k$ th copy of $Z$. Since the elements $(0, \ldots, 0,1,0, \ldots, 0)$ must each be mapped onto nontrivial elements of $Z_{p}$, it follows that the only cases where there is precisely one admissible mapping is if either $p=2$ or $\mu=1$. This completes the proof of Lemma 5 .

Proof of Theorem 3. Let $K$ be a closed $b$-braid knot, $\left({ }^{5}\right)$ and let $p$ be any integer $\geqslant 2$. By the argument used in the proof of Theorem 2, we may always represent $\left(S^{3}, K\right)$ as

$$
\left(S^{3}, K\right)=\left(D, A_{b}\right) \cup_{\varphi}\left(D^{\prime}, A_{b}^{\prime}\right)
$$

for some autohomeomorphism $\varphi:\left(\partial D-\partial A_{b}\right) \rightarrow\left(\partial D-\partial A_{b}\right)$. We may then reverse the entire argument used in the proof of Theorem 2 to construct the $p$-fold cyclic covering of $D$ (respectively $D^{\prime}$ ) branched over $A_{b}$ (respectively $A_{b}^{\prime}$ ). This covering space will be a handlebody $Y_{b, p}$ (respectively $Y_{b, p}^{\prime}$ ) of genus $g=$ $(b-1)(p-1)$.

Since $K$ is a closed $b$-braid, the condition of Lemma 4 will be satisfied, hence the homeomorphism $\varphi$ lifts to a homeomorphism $\Phi$ of $\partial Y_{b, p}$. The

(5) The reader is referred to $\S 2$ for the definition of a closed $b$-braid. Note that (by Lemmas 1 and 2) every closed $b$-braid is also a $2 b$-plat, and hence has bridge number $<b$; however the converse need not be true. 
homeomorphism $\Phi$ may be used to construct a 3-manifold $M=Y_{b, p} U_{\tau \Phi} Y_{b, p}^{\prime}$, which is now seen to be a $p$-symmetric Heegaard splitting of genus $\boldsymbol{g}=$ $(b-1)(p-1)$. Also, $M$ is a $p$-fold cyclic covering space of $S^{3}$, branched over $K$. By Lemma 5 , this covering space is unique. This completes the proof of Theorem 3.

Our next result, Theorem 4, is a sharpened version of Theorems 2 and 3, which holds in the special case $p=2$.

Proof of Theorem 4. To establish Theorem 4, we must prove

(4.1) Every closed orientable 3-manifold of 2-symmetric Heegaard genus $\boldsymbol{g}$ is a 2 -fold covering of $S^{3}$ branched over a link of at most $g+1$ bridges.

(4.2) Every 2-fold covering of $S^{3}$ branched over a link of $g+1$ bridges has 2-symmetric Heegaard genus at most $g$.

Statement (4.1) is precisely Theorem 2 , for the special case $p=2$. Statement (4.2) is a strong version of Theorem 3, which holds when $p=2$. The proof of statement (4.2) may be accomplished by a sequence of steps just like those used to prove Theorem 3, except that

(a) If $p=2$, Lemma 4 tells us that every autohomeomorphism $\varphi$ of ( $\partial D-$ $\left.\partial A_{b}\right)$ lifts to $\partial Y_{b, p}$. Hence we may consider all $(g+1)$-bridge links, instead of restricting our attention to the sublcass of closed $(g+1)$-braids.

(b) If $p=2$, the statement of Lemma 5 allows us to consider links of $\mu \geqslant 1$ components, instead of restricting our attention to the case $\mu=1$, i.e. to proper knots.

This completes the proof of Theorem 4.

Proof of Theorem 5. Theorem 5 will follow immediately from Theorem 4 , if we can show that every closed, orientable 3-manifold of Heegaard genus $g=1$ or 2 also has 2 -symmetric Heegaard genus $g$. This is true because every homeomorphism $\Phi$ of the boundary $\partial Y_{g}$ of a handlebody of genus $g \leqslant 2$ is isotopic to a homeomorphism which commutes with a particular involution $T: \partial Y_{\boldsymbol{g}}$ $\rightarrow \partial Y_{g}$, which is such that $\partial Y_{g} / T$ is a 2 -sphere. To verify this latter statement, note that, by Lemma 3 of $\S 3$, every homeomorphism $\Phi$ of $\partial Y_{g}$ is isotopic to a product of the Lickorish twists $t_{c_{1}}, \ldots, t_{c_{3 g-1}}$, and that if $g \leqslant 2$ each such twist may be chosen to commute with a rotation $T$ of period 2 about the $x$ axis in Figure 4. $\left({ }^{6}\right)$ Since the quotient space $\partial Y_{g} / T$ is a 2-sphere in this case, the proof of Theorem 5 is complete.

Finally we approach the proof of Theorem 1. Statement (1.1) of Theorem 1 , which relates to the case of $g \leqslant 2$, is contained in Theorem 5 , hence we may restrict our attention to the case $g \geqslant 3$. Our proof will be, essentially, an escalation of the technique used in the proof of Theorems 2 and 3, however in the

(6) The special symbol $T$ will be used instead of $P$ to refer to this particular rotation. 
general case to be considered now the Heegaard splitting $Y_{2} U_{\tau \Phi} Y_{g}^{\prime}$ is not assumed to be $p$-symmetric, and the covering space projection we will define when we develop a representation of $M=Y_{g} \cup_{\tau \Phi} Y_{g}^{\prime}$ as a branched covering of $S^{3}$ will not be a regular covering space.

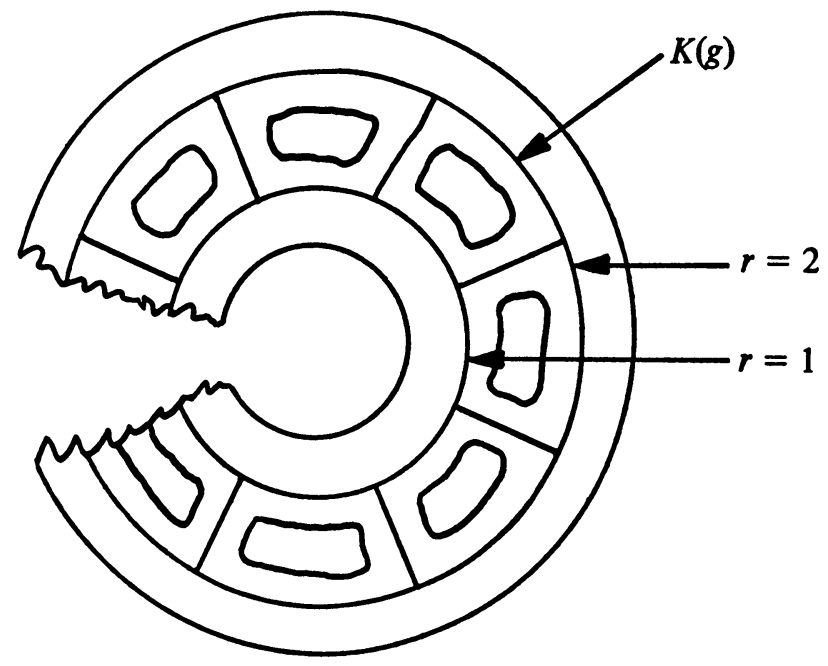

FIgURE 6. Projection of $Y_{g}$ onto the plane $z=0$

Proof of Theorem 1. As before, let $(r, \theta, z)$ denote cylindrical coordinates in $E^{3}$. The reader is referred to Figure 6 . Let $K(g), g \geqslant 3$, be the union of the line segments $\{[(1,(2 k+1) \pi /(g-1), 0),(2,(2 k+1) \pi /(g-1), 0)] ; k=$ $0, \ldots, g-2\}$ and the circles $z=0, r=1$ and $z=0, r=2$. Let $Y_{g}$ be a regular neighborhood of $K(g)$ which is invariant under a rotation $L$ of $2 \pi /(g-1)$ radians about the $z$ axis, and also under a rotation $B_{k}$ of $\pi$ radians about the lines $z=0$, $\theta=(2 k+1) \pi /(g-1)$, for each $k=0, \ldots, g-2$. We also assume that the intersection of $Y_{g}$ with each half-plane $\theta=\theta_{0}$ has central symmetry, and we denote the homeomorphism of $Y_{g}$ obtained by simultaneously performing the central symmetry on each such section by $M$.

Note that $M$ commutes with $L$ and also with $B_{0}, \ldots, B_{g-2}$. Thus we have defined an action on $Y_{g}$ by the finite group $G$ generated by $L, M$ and $B_{0}$, $\ldots, B_{g-2}$. The group $G$ is an extension of order 2 of a dihedral group of order $2(g-1)$. Nontrivial elements of $G$ with fixed points are $\left\{M, B_{k}\right.$ and $M B_{k}, k=$ $0, \ldots, g-2\}$. The fixed point sets of $M, B_{k}$ and $M B_{k}$ are the intersection of $Y_{g}$ with the circle $z=0, r=1 \frac{1}{2}$; the line $z=0, \theta=2 k \pi /(g-1)$, and the line $r=1 \frac{1}{2}, \theta=2 k \pi /(g-1)$ respectively. (See Figure 7.)

Let $T_{g}=\partial Y_{g}$. The group $G$ acts on $T_{g}$ by restriction. Let $a_{i}, b_{i}, c_{i}, d_{i}$ and $e$ be the curves on $T_{g}$ depicted in Figure 7. Let $\alpha_{i}, \beta_{i}, \gamma_{i}, \delta_{i}$ and $\epsilon$ be the Dehn twists about these curves (see $\S 3$ for the definition of a Dehn twist). We 


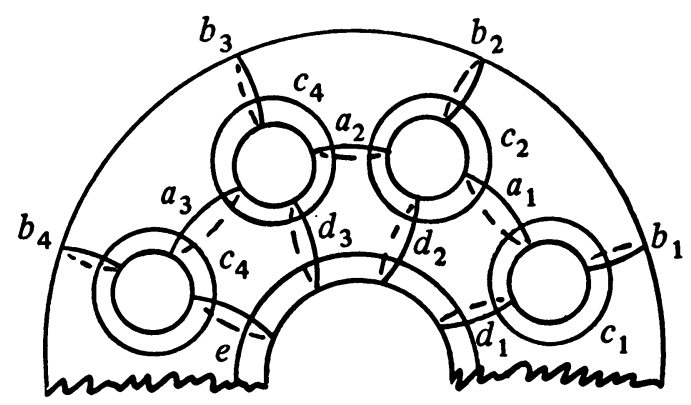

FIGURE 7. Curves on $T_{g}=\partial Y_{g}$

may choose these twists and curves in such a way that $\alpha_{k} M=M \alpha_{k}, \beta_{k} B_{k}=B_{k} \beta_{k}$, $\gamma_{k} B_{k}=B_{k} \gamma_{k}, \delta_{k} B_{k}=B_{k} \delta_{k}$ and $\epsilon B_{k}=B_{k} \epsilon$ for each $k=0, \ldots, g-2$. By Lemma 3, this collection of Dehn twists generates the mapping class group of $T_{\boldsymbol{g}}$.

LEMMA 6. Given any Dehn twist $\omega$ in the above collection, there is an element $\chi(\omega) \in G$ such that:

(i) $\omega$ commutes with $\chi(\omega)$.

(ii) $\chi(\omega)=B_{k}$ or $\chi(\omega)=M ; k=0, \ldots, g-2$.

(iii) If $\underline{\omega}$ is the projection of $\omega$ to the manifold $T_{g} / \chi(\omega)$, then $\underline{\omega}$ is isotopic to the identity map.

(iv) Let $P(\omega)$ be the image of the fixed point set of $\chi(\omega)$ under the mapping $\pi: T_{g} \rightarrow T_{g} / \chi(\omega)$. Let $Q(\omega)$ be the image under $\pi$ of the remaining fixed points. Then the isotopy $\underline{\omega}_{t}$ of $\omega$ may be chosen so that $P \cap \underline{\omega}_{t}(Q)=\varnothing$ for all $0 \leqslant t \leqslant 1$.

Proof of Lemma 6. If $\omega=\beta_{k}, \gamma_{k}$ or $\delta_{k}$ choose $\chi(\omega)=B_{k}$. If $\omega=\alpha_{k}$, choose $\chi(\omega)=M$. If $\omega=\epsilon$ choose $\chi(\omega)=B_{k}$ for any $k$. Conditions (i) and (ii) above are satisfied. In each case the support of $\underline{\omega}$ on $T_{g} / \chi(\omega)$ is a disc, hence (iii) is also satisfied.

If $\omega=\beta_{i}$ or $\delta_{i}$, the disc supporting $\underline{\omega}$ contains no points of $Q(\omega)$ at all. If $\omega=\gamma_{i}$ or $\alpha_{i}$, then there is only one point of $Q(\omega)$ in the disc supporting $\underline{\omega}$. In fact $\underline{\omega}$ is a twist centered at this point. In either of these cases $\underline{\omega}$ can be untwisted without moving the points of $Q(\underline{\omega})$ at all, and (iv) is easily satisfied. Finally, if $\omega=\epsilon$, then $\underline{\omega}$ is a twist exchanging a pair of points of $P(\omega)$. The line segment connecting these points contains all the points of $Q(\omega)$ in the support of $\underline{\omega}$ and is left invariant as a set by $\underline{\omega}$ (the pre-image of this line segment is the support of $\epsilon$ intersected with the $x-y$ plane). As $\underline{\omega}$ is untwisted, $\underline{\omega}_{t}(Q(\omega))$ traces out the path indicated in Figure 8, easily avoiding the set $P(\omega)$. This completes the proof of Lemma 6. 


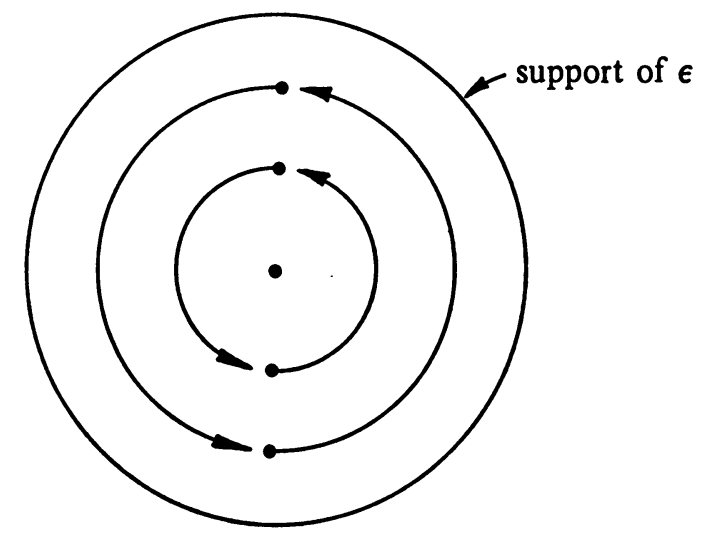

FIGURE 8. Effect of $\epsilon_{t}$ on points in $Q(\epsilon)$

Continuing the proof of Theorem 1, let $Y_{g}^{\prime}$ be another copy of $Y_{g}$. Let $\tau$ be the map that identifies $Y_{g}$ with $Y_{g}^{\prime}$. Let $T_{g}^{\prime}=\tau\left(T_{g}\right)=\tau\left(\partial Y_{g}\right)=\partial Y_{g}^{\prime}$. In what follows we shall allow the group $G$ to act on the sets $T_{g}^{\prime}$ and $Y_{g}^{\prime}$, and allow the Dehn twists to act on $T_{g} \times\{n+1\}$, considered as a subset of the set $T_{g} \times$ $[n, n+1]$. This is always done in the obvious way.

Let $\tau_{j}: T_{g} \times\{2 j-1\} \rightarrow T_{g} \times\{2 j\}$ be defined by $\tau_{j}(z, 2 j-1)=(z, 2 j)$. Let $\omega_{j}$ be a Dehn twist acting on $T_{g} \times\{2 j-1\}$. We will use the symbol $T_{g} \times$ $[2 j-2,2 j-1] \cup_{\tau_{j} \omega_{j}} T_{g} \times[2 j, 2 j+1]$ to indicate that $T_{g} \times\{2 j-1\}$ is to be identified with $T_{g} \times\{2 j\}$ by the rule $\left(\omega_{j}(z), 2 j-1\right)=(z, 2 j)$.

LEMma 7. Every closed, orientable 3-manifold $M$ of Heegaard genus $g$ admits a representation of the form:

$$
\begin{aligned}
M= & Y_{g} U_{\tau_{0}} T_{g} \times[0,1] U_{\tau_{1} \omega_{1}} T_{g} \times[2,3] U_{\tau_{2} \omega_{2}} \cdots \\
& U_{\tau_{m} \omega_{m}} T_{g} \times[2 m, 2 m+1] U_{\tau_{m+1}} Y_{g}^{\prime}
\end{aligned}
$$

where $\tau_{0}$ and $\tau_{m+1}$ are the obvious identifications of $\partial Y_{g}=T_{g}$ with $T_{g} \times\{0\}$ and of $T_{\boldsymbol{g}} \times\{2 m+1\}$ with $T_{\boldsymbol{g}}^{\prime}=\partial Y_{\boldsymbol{g}}^{\prime}$.

Proof of Lemma 7. Since the Dehn twists generate the mapping class group, $M$ has Heegaard genus $g$, and $T_{g}$ has a collar neighborhood, the Heegaard splitting of $M$ yields the representation

$$
\begin{aligned}
M= & Y_{g} U_{\tau_{0}} T_{g} \times[0,1] U_{\tau_{1}} T_{g} \times[2,3] \cup \ldots \\
& U_{\tau_{m}} T_{g} \times[2 m, 2 m+1] U_{\tau_{m+1}} \omega_{m} \ldots \omega_{2} \omega_{1} Y_{g}^{\prime} .
\end{aligned}
$$

A homeomorphism between $M$ and a manifold $M^{\prime}$ with the required form is defined below: 


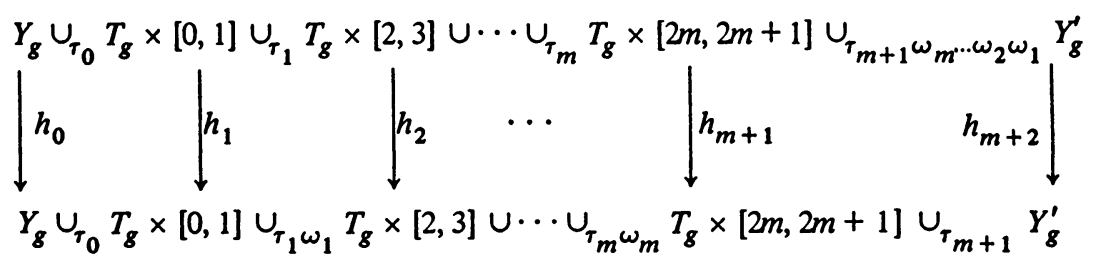

Define $h_{0}(z)=z, h_{m+2}(z)=z, h_{1}(z, t)=(z, t)$ and $h_{j}(z, t)=\left(\omega_{j-1} \cdots\right.$ $\left.\omega_{2} \omega_{1}(z), t\right)$ for each $j=2, \ldots, m+1$. This proves Lemma 7 .

Before proving the next lemma we introduce a definition which we will make use of only in the remainder of this section. Given a branched covering space projection $\pi: M \rightarrow N$, where $M$ and $N$ are compact manifolds, a point $x \in$ $M$ is called ordinary if $p$ is a local homeomorphism in some neighborhood of $x$. A point that is not ordinary is called irregular, and the set of irregular points is called the irregular set. Notice that the branch cover is precisely the inverse image of the image under $\pi$ of the irregular set.

The next lemma is a weak version of Theorem 1 .

LEMMA 8. Let $M$ be defined by equation (5). Then there is a branched covering $\pi: M \rightarrow S^{3}$, where $S^{3}$ is represented by

$$
\begin{aligned}
S^{3}= & \left(Y_{g} / G\right) \cup_{\tau_{0}}\left(T_{g} / G\right) \times[0,1] \cup_{\tau_{1}}\left(T_{g} / G\right) \times[2,3] \cdots \\
& \cup_{\tau_{m}}\left(T_{g} / G\right) \times[2 m, 2 m+1] \cup_{\tau_{m+1}}\left(Y_{g}^{\prime} / G\right)
\end{aligned}
$$

such that

(i) $\pi$ restricted to $Y_{g} \cup Y_{g}^{\prime} \cup T_{g} \times[2 m, 2 m+1] \cup T_{g} \times\{0,2,4, \ldots$, $2 m\}$ is the natural projection.

(ii) $\pi:\left(T_{g}\right) \times[2 j, 2 j+1] \rightarrow\left(T_{g} / G\right) \times[2 j, 2 j+1]$ preserves the second coordinate $j=0, \ldots, m$.

(iii) The irregular set is a 1-complex. The singular points of this 1-complex are the points $\left(1 \frac{1}{2},(2 k+1) \pi /(g-1), 0\right)$ and $\tau\left(1 \frac{1}{2},(2 k+1) \pi /(g-1), 0\right)$ in $Y_{g}$ and $Y_{g}^{\prime}$ respectively.

Proof of Lemma 8. First note that $\left(T_{g} / G\right)$ and $\left(Y_{g} / G\right)$ are $S^{2}$ and $D^{3}$ respectively. To see this observe that a fundamental set for the action of $G$ on $Y_{g}$ is $Y_{g} \cap\{(r, \theta, z) \mid 0 \leqslant r \leqslant 112,0 \leqslant \theta \leqslant \pi /(g-1)\} .\left(Y_{g} / G\right)$ is obtained from the fundamental set by making the identifications $(r, \theta, z)=(r, \theta,-z)$ for points $(r, \theta, z)$ lying in the half-planes $\theta=0$ or $\theta=\pi /(g-1)$ or the cylinder $r=1 \frac{1}{2}$ (see Figure 9).

Next, we use condition (i) to define $\pi$ on the set denoted in (i). We wish to extend the definition of $\pi$ to the rest of $M$ and then check that conditions (ii) and (iii) are satisfied. Pick some $j: 0 \leqslant j \leqslant m-1$. We wish to extend the 


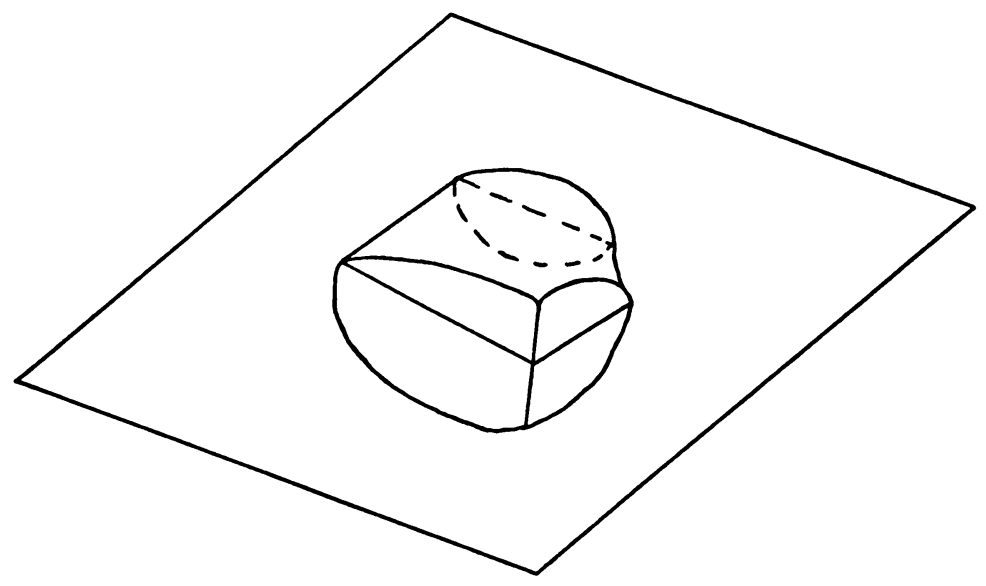

FIGURE 9. Fundamental set for action of $G$ on $Y_{g}$

definition of $\pi$ from $T_{g} \times\{2 j\} \cup T_{g} \times\{2 j+2\}$ to $T_{g} \times[2 j, 2 j+1] \cup_{\tau_{j+1}} \omega_{j+1}$ $T_{g} \times\{2 j+2\}$. Let $\chi\left(\omega_{j+1}\right) \in G$ be the element whose existence is guaranteed by Lemma 6 . Let $\underline{\omega}_{j+1, t}$ be the isotopy whose existence is guaranteed by Lemma 6, parts (iii) and (iv) $\left(\underline{\omega}_{j+1,0}=\mathrm{id} ; \underline{\omega}_{j+1,1}=\underline{\omega}_{j+1}\right)$. Let

$$
\begin{aligned}
p_{j}: & T_{g} \times[2 j, 2 j+1] \cup_{\tau_{j+1} \omega_{j+1}} T_{g} \times\{2 j+2\} \\
& \rightarrow\left(T_{g} /\left(\chi\left(\omega_{j+1}\right)\right)\right) \times[2 j, 2 j+1] \cup_{\tau_{j+1} \underline{\omega}_{j+1}}\left(T_{g} /\left(\chi\left(\omega_{j+1}\right)\right)\right) \times\{2 j+2\}
\end{aligned}
$$

be the natural projection. Let

$$
\begin{aligned}
q_{j} & :\left(T_{g} / \chi\left(\omega_{j+1}\right)\right) \times[2 j, 2 j+1] \cup_{\tau_{j+1} \underline{\omega}_{j+1}}\left(T_{g} / \chi\left(\omega_{j}\right)\right) \times\{2 j+2\} \\
& \rightarrow\left(T_{g} / \dot{\chi}\left(\omega_{j+1}\right)\right) \times[2 j, 2 j+1] \cup_{\tau_{j+1}}\left(T_{g} / \chi\left(\omega_{j+1}\right)\right) \times\{2 j+2\}
\end{aligned}
$$

be the homeomorphism $(x, t) \rightarrow\left(\underline{\omega}_{j+1, t-2 j}(x), t\right), 2 j \leqslant t \leqslant 2 j+1$, and $(x$, $2 j+2) \rightarrow(x, 2 j+2)$. Note that $q_{j}$ is well defined and that $q_{j}$ restricted to $T_{\boldsymbol{g}} \times\{2 j, 2 j+1\}$ is the identity. Let

$$
\begin{aligned}
\gamma_{j} & :\left(T_{g} / \chi\left(\omega_{j+1}\right)\right) \times[2 j, 2 j+1] \cup_{\tau_{j+1}}\left(T_{g} / \chi\left(\omega_{j+1}\right)\right) \times\{2 j+2\} \\
& \rightarrow\left(T_{g} / G\right) \times[2 j, 2 j+1] \cup_{\tau_{j+1}}\left(T_{g} / G\right) \times\{2 j+2\}
\end{aligned}
$$

be the natural projection. Now define $\pi$ on $T_{g} \times[2 j, 2 j+1] \cup T_{g} \times\{2 j,+2\}$ to be $\gamma_{j} q_{j} p_{j}$ for each $j$, and note that $\pi$ is well defined because $\gamma_{j} q_{j} p_{j}$ is just the natural projection on the sets $T_{g} \times\{2 j, 2 j+2\}$.

Now we investigate the irregular set of $\pi$ restricted to $T_{g} \times[2 j, 2 j+1]$. Since it is easily checked that $p_{j}, q_{j}$, and $\gamma_{j}$ are all branched coverings, this set is equal to $P_{j} \cup p_{j}^{-1} q_{j}^{-1} Q_{j}$ where $P_{j}$ is the irregular set of the natural projection $p_{j}: T_{g} \times[2 j, 2 j+1] \rightarrow\left(T_{g} / \chi\left(\omega_{j}\right)\right) \times[2 j, 2 j+1]$ and $Q_{j}$ is the irregular set of 
the natural projection

$$
\gamma_{j}:\left(T_{g} / \chi\left(\omega_{j}\right)\right) \times[2 j, 2 j+1] \rightarrow\left(T_{g} / G\right) \times[2 j, 2 j+1]
$$

By the definition of $q_{j}$ (utilizing part (iv) of Lemma 6) $P_{j}$ and $p_{j}^{-1} q_{j}^{-1} Q_{j}$ are disjoint sets. Thus the irregular set consists of $8(g-1)$ arcs, whose intersection with the set $T_{g} \times\{t\}$ consists of $8(g-1)$ points. Putting together all the irregular sets of $\pi$ restricted to $T_{g} \times[2 j, 2 j+1]$ we see that the irregular set of $\pi$ restricted to $T_{g} \times[0,1] \cup_{\tau_{1} \omega_{1}} T_{g} \times[2,3] \cup \cdots \cup_{\tau_{m} \omega_{m}} T_{g} \times[2 m, 2 m+1]$ consists of $8(g-1)$ disjoint arcs. Thus all the singular points of the irregular set belong to the set $Y_{g} \cup Y_{g}^{\prime}$. Checking the definition of $\pi$ on these sets $Y_{g} \cup Y_{g}^{\prime}$ we see that the singular points of the irregular set are the points $\left(1 \frac{1}{2},(2 k+1) \pi /(g-1), 0\right)$ and $\tau\left(1 \frac{1}{2},(2 k+1) \pi /(g-1), 0\right), k=0, \ldots, g-2$. This completes the proof of Lemma 8.

We may now complete the proof of Theorem 1, part (1.2). First we eliminate the singular point from the irregular set. In view of Lemma 8 , and the definition of $\pi$, all we need to do is to show that we can modify the map $\pi$ of Lemma 8 in a neighborhood of a point $\left(1 \frac{1}{2},(2 k+1) \pi /(g-1), 0\right)$ so as to eliminate the singular point.

Let $H$ be the subgroup of $G$ consisting of elements that fix the point $\left(1 \frac{1}{2},(2 k+1) \pi /(g-1), 0\right)$. Let $\eta_{1}: Y_{g} \rightarrow\left(Y_{g} / M\right), \eta_{2}:\left(Y_{g} / M\right) \rightarrow\left(Y_{g} / H\right)$ and $\eta_{3}:\left(Y_{g} / H\right) \rightarrow\left(Y_{g} / G\right)$ be the natural projections. Then $\pi$ restricted to $Y_{g}$ equals $\eta_{3} \eta_{2} \eta_{1}$. The irregular set of $\eta_{1}$, denoted $B_{1}$, is the circle $(z=0, r=11 / 2) \cap Y_{g}$. The irregular set of $\eta_{2}$, denoted $B_{2}$, is $\eta_{1}$ (the ray $(z=0, \theta=(2 k+1) \pi /(g-1) \cap$ $\left.Y_{g}\right)$ ). The map $\eta_{3}$ is a local homeomorphism in a neighborhood of $\eta_{2} \eta_{1}\left(1 \frac{1}{2},(2 k+1) \pi /(g-1), 0\right)$. Let $\psi$ be a homeomorphism of $Y_{g} / M$ supported in a small ball $D$ centered at $\eta_{1}\left(1 \frac{1}{2},(2 k+1) \pi /(g-1), 0\right)$ such that $\psi\left(\eta_{1}\left(B_{1}\right)\right) \cap$ $B_{2} \cap D=\varnothing$. The map $\eta_{3} \eta_{2} \psi \eta_{1}$ has no singular points in its irregular set and agrees with $\pi$ except in a small neighborhood of $\left(1 \frac{1}{2},(2 k+1) \pi /(g-1), 0\right)$. We can repeat the process at each of the points $\left(1 \frac{1}{2},(2 k+1) \pi /(g-1), 0\right)$ and $\tau(11 / 2,(2 k+1) \pi /(g-1), 0)$ until all singular points have been eliminated from the irregular set of $\pi$.

The irregular set, which we shall call $S$, is now a 1-manifold, but its image has singular points. To eliminate these we shall further modify the map $\pi$ so that $\pi$ restricted to $S$ is an embedding.

Let $M$ be the set of multiple points of $S$, i.e. $M=\{x \in S \mid \pi(y)=\pi(x)$ for some $y \in S$ with $y \neq x$. Let $U_{i}, i=1, \ldots, n$, be a covering of $\pi(S)$ by open balls such that the restriction of $\pi$. to any component of $\pi^{-1}\left(U_{i}\right)$ embeds the intersection of $S$ with that component. Suppose $V$ is a component of $\pi^{-1}\left(U_{i}\right)$, for some $i$, such that $V \cap M \neq \varnothing$. Let $\psi$ be a homeomorphism of $S^{3}$ supported on $U_{i}$ such that $\psi(\pi(V \cap S)) \cap \pi(S)=\varnothing$. (Such a homeomorphism exists by a 
general position argument.) Now define $\pi^{\prime}(x)=\pi(x)$ if $x \notin V$ and $\pi^{\prime}(x)=$ $\psi(\pi(x))$ if $x \in V$.

The branched covering $\pi^{\prime}$ has the following properties: (1) For any $i, i=$ $1, \ldots, n, \pi^{\prime-1}\left(U_{i}\right)=\pi^{-1}\left(U_{i}\right)$. (2) The set of multiple points for $\pi^{\prime}, M^{\prime}$ is contained in $M$. (3) $V \cap M^{\prime}=\varnothing$, but $V \cap M \neq \varnothing$. We can iterate this process until no component of the inverse image of any $U_{i}$ contains a multiple point. The. final branched covering we obtain, $\tilde{\pi}$, embeds the irregular set. Thus the branch set $\tilde{\pi}(S)$ is a one-manifold. Since $\tilde{\pi}$ is a local homeomorphism on the complement of $S$, the branch cover $\tilde{\pi}^{-1} \tilde{\pi}(S)$ is also a 1 -manifold.

5. An algorithm to decide whether a 3-manifold which admits a 2-symmetric Heegaard splitting is $S^{3}$. In this section we apply the results of $\S 4$ to establish that there is an effective algorithm to decide whether a 3-manifold which admits a 2-symmetric Heegaard splitting is $S^{3}$. The reader is reminded that this class includes all 3-manifolds of Heegaard genus $g \leqslant 2$. This result was established earlier by the authors in [8]. As an application, we will use our algorithm to give a new and very simple proof that every genus 1 homology sphere is $S^{3}$.

Let $Y_{g}$ be a handlebody of genus $g$ which is imbedded in 3-space in the manner indicated in Figure 4, so that it is invariant under a rotation $T$ of $180^{\circ}$ about the $z$ axis. Note that the orbit space $Y_{g} / T$ is a 3-ball $D$, and that $T$ has period $p=2$. Let $Y_{g}^{\prime}$ be a second copy of $Y_{g}$, and let $\tau: Y_{g} \rightarrow Y_{g}^{\prime}$ be the natural map. Let $\Phi: \partial Y_{g} \rightarrow \partial Y_{g}$ be a homeomorphism which commutes with the restriction of $T$ to $\partial Y_{g}$, and let $M=Y_{g} \cup_{\tau \Phi} Y_{g}^{\prime}$ be the 3-manifold defined by using the rule in equation (1) to identify points on $\partial Y_{g}$ and $\partial Y_{g}^{\prime}$. Thus $M$ is defined by the 2-symmetric Heegaard splitting $Y_{g} \cup_{\tau \Phi} Y_{g}^{\prime}$. Recall that every 3 manifold of Heegaard genus $g \leqslant 2$ admits such a representation, because by Lemma 3 every homeomorphism $\Phi$ of $\partial Y_{g}$ is isotopic to a product of Lickorish twists $t_{c_{1}}, \ldots, t_{c_{3 g-1}}$ about the curves $c_{1}, \ldots, c_{3 g-1}$ in Figure 4 , and if $g \leqslant 2$ each such twist may be defined in such a way that it commutes with $T$. If $g \geqslant 3$, we restrict ourselves to twists about $c_{1}, \ldots, c_{2 g+1}$.

Let $[\Phi]$ denote the isotopy class of $\Phi$. We will assume that $[\Phi]$ is defined as a product of twists, i.e., that we are given

$$
[\Phi]=\left[t_{c_{\mu_{r}}}^{\epsilon_{r}}\right] \cdots\left[t_{c_{\mu_{2}}}^{\epsilon_{2}}\right]\left[t_{c_{\mu_{1}}}^{\epsilon_{1}}\right], \quad \epsilon_{i}= \pm 1, \mu_{i}=1, \ldots, 2 g+1 .
$$

The algorithm. Let $[\Phi]$ be given by equation (6).

1. Construct the $(2 g+2)$-string braid

$$
\beta=\sigma_{\mu_{r}}^{\epsilon_{r}} \cdots \sigma_{\mu_{1}}^{\epsilon_{1}}
$$

where $\sigma_{i}(i=1, \ldots, 2 g+1)$ is a standard generator of the $(2 g+2)$-string Artin braid group. [See Figure 1.] 
2. Construct the $(2 g+2)$-plat $L$ determined by the geometric braid $\beta$ (see $\S 2)$.

3. Apply the algorithm given by Haken in [16], or by Schubert in [31] to decide whether $L$ is the trivial knot type.

We assert:

ThEOREM 6. The 2-symmetric 3-manifold $M=Y_{g} U_{\tau \Phi} Y_{g}^{\prime}$ is $S^{3}$ if and only if $L$ defines the trivial knot type.

Proof of Theorem 6. Suppose that $M$ is homeomorphic to $S^{3}$. Then $T$ is an involution of the 3-sphere, hence by a theorem of Waldhausen [33] the fixed point set $F$ of $T$ is an unknotted circle. Hence its image $\pi(F)$ under the collapsing map $\pi: M \rightarrow M / T$ is also the trivial knot type. We claim that $\pi(F)$ is precisely the plat defined by the $(2 g+2)$-braid $\beta$ in equation (7). If we can establish this, it will follow that a necessary condition for $M \sim S^{3}$ is that this plat be trivial. To see that this condition is also sufficient, note that if $\pi(F)$ is the trivial knot type, then by the construction described in the proof of Theorem 2, $\S 4, M$ is the 2 -fold cyclic covering of

$$
M / T=\left(Y_{g} \cup_{\tau \Phi} Y_{g}^{\prime}\right) / T=D \cup_{\tau \varphi} D^{\prime} \sim S^{3}
$$

branched over the trivial knot. But then $M$ can only be $S^{3}$, hence the condition is also sufficient.

It remains to establish that $\pi(F)$ is the plat defined by the $(2 g+2)$-string braid $\beta$. To see this, recall that it was established in the proof of Theorem 2 that $\pi(F)$ is the point set $A_{b} \cup_{\tau \varphi} A_{b}^{\prime} \subset D \cup_{\tau \varphi} D^{\prime}$, where $A_{b}$ is a collection of $b=$ $2 g+2$ unknotted arcs which are contained in the 3-ball $D$, and $A_{b}^{\prime}=\tau\left(A_{b}\right)$ is the corresponding collection in $D^{\prime}=\tau(D)$ (cf. Figure 5); also that $D \cup_{\tau \varphi} D^{\prime}$ is $S^{3}$ (the 3-balls $D$ and $D^{\prime}$ are sewn together by the surface mapping $\tau \varphi: \partial D \rightarrow$ $\partial D^{\prime}$, where $\left.\varphi=\pi \Phi \pi^{-1}\right)$. The mapping $\Phi: \partial Y_{g} \rightarrow \partial Y_{g}$ is the twist product given in equation (6), hence $[\varphi]$ will be the corresponding product of twists $\left[\pi_{c_{\mu_{i}}{ }_{i}}^{{ }^{2}} \pi^{-1}\right]$, and $\left[\pi t_{c_{\mu}} \pi^{-1}\right]$ is precisely the elementary braid autohomeomorphism $\sigma_{i}$ of the surface $\left(\partial D-\partial A_{b}\right)$, as defined in $\S 2 .\left({ }^{7}\right)$ Thus $\Phi$ projects to

$$
[\varphi]=\beta=\sigma_{\mu_{r}}^{\epsilon_{r}} \cdots \sigma_{\mu_{2}}^{\epsilon_{2}} \sigma_{\mu_{1}}^{\epsilon_{1}}
$$

hence $\pi(F)$ is precisely the plat determined by $\beta .\left({ }^{8}\right)$ This proves Theorem 6 .

(7) The fact that $\left[\pi t_{c_{i}} \pi^{-1}\right]=\sigma_{i}$ is clear from the definitions of the twists $t_{c_{i}}$ and $\sigma_{i}$ and the projection $\pi$. For an explicit proof, see [6, Chapter 4].

(8) Note that we have followed the convention that mappings are applied from right to left. Thus $\sigma_{\mu_{r}} \cdots \sigma_{\mu_{1}}$ means "apply $\sigma_{\mu_{1}}^{\epsilon_{1}}$ first, then $\sigma_{\mu_{2}}, \cdots \cdots$. This convention is opposite to the convention normally followed by group theorists, e.g., as in [20, pp. 173179]. However, since we are not concerned with orientations, it will not matter whether we construct our plat from the braid $\sigma_{\mu_{1}}^{\epsilon_{1}} \cdots \sigma_{\mu_{r}}$ or from the braid $\sigma_{\mu_{r}}^{\epsilon_{r}} \cdots \sigma_{\mu_{1}}^{\epsilon_{1}}$, as one may be obtained from the other by turning the braid diagram upside down and over. 
REMARK. We were unable to generalize our algorithm to the case of 3 manifolds which are represented by $p$-symmetric Heegaard splittings, where $p>$ 2 , because the "Smith conjecture" [34] for periods $p>2$ has not been established. If this gap were filled, our algorithm could be adapted to an arbitrary $p$ symmetric Heegaard splitting. By a recent result of Montesinos [24] this would still be a proper subset of all closed orientable 3-manifolds.

As an application of our algorithm, we now give a new and very brief proof of a classical result: every genus 1 homology 3-sphere is $S^{3}$. The algorithm will also be applied later (in \$7) to the more difficult cases of Heegaard splittings of genus 2 .

COROLlary 1. Let $M=Y_{1} \cup_{\tau \Phi} Y_{1}^{\prime}$ be a 3.manifold which is defined by a genus 1 Heegaard splitting. Then $M \sim S^{3}$ if and only if $H_{1}(M)=0$.

Proof OF COROLlary 1. Let $a, b$ (respectively $a^{\prime}, b^{\prime}$ ) be generators of $\pi_{1} \partial Y_{1}$ (respectively $\pi_{1} \partial Y_{1}^{\prime}$ ), with $\tau(a)=a^{\prime}, \tau(b)=b^{\prime}$. Suppose that $\Phi: \partial Y_{1} \rightarrow$ $\partial Y_{1}$ has the action:

$$
[\Phi]: a \rightarrow a^{n} 11 b^{n} 12,
$$

$$
b \rightarrow a^{n_{21}} b^{n_{22}}, \quad n_{11} n_{22}-n_{12} n_{21}=1 .
$$

Suppose also that the natural inclusion maps from $\pi_{1} \partial Y_{1} \rightarrow \pi_{1} Y_{1}$ (respectively $\pi \partial Y_{1}^{\prime} \rightarrow \pi_{1} Y_{1}^{\prime}$ ) have kernels generated by $b$ (respectively $\left.b^{\prime}\right)$. With these conventions, a straightforward application of van Kampen's theorem shows that $\pi_{1} M$ admits the presentation:

$$
\left\langle a ; a^{n} 21=1\right\rangle .
$$

Thus $H_{1}(M)=0$ if and only if $n_{21}= \pm 1$.

Our proof will be complete if we can show that $n_{21}= \pm 1$ implies $M \sim S^{3}$.

Let $t_{c_{1}}, t_{c_{2}}$ be Dehn twists about representatives of $b \simeq c_{1}, a \simeq c_{2}$ respectively (cf. Figure 2). These twists induce the automorphisms

$$
\begin{aligned}
{\left[t_{c_{1 *}}\right]: a } & \rightarrow a b^{-1}, & {\left[t_{c_{2^{*}}}\right]: a } & \rightarrow a, \\
b & \rightarrow b, & b & \rightarrow a b .
\end{aligned}
$$

An easy calculation shows that:

$$
[\Phi]=\left[t_{c_{1}}\right]^{n_{22}-1}\left[t_{c_{2}}\right]^{n_{21}}\left[t_{c_{1}}\right]^{1-n_{11}}, \quad n_{21}= \pm 1 .
$$

We may now apply our algorithm to decide whether $M \sim S^{3}$. To accomplish this, replace the map $\Phi$ by the 4-braid

$$
\beta=\sigma_{1}^{n_{22}-1} \sigma_{2}^{n} 21 \sigma_{1}^{1-n_{11}}, \quad n_{21}= \pm 1,
$$


and construct the 4-plat determined by $\beta$ (see Figure 10). It is immediate that this plat defines the trivial knot type for every choice of the integers $n_{11}, n_{22}$, hence $M \sim S^{3}$.

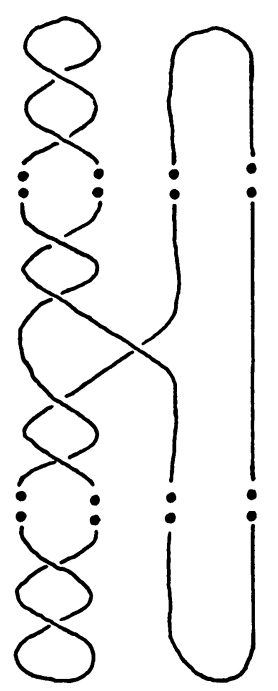

FIGURE 10. 4-plat corresponding to the genus 1 Heegaard splittings $\Phi=t_{c_{1}}^{n_{22}-1} t_{c_{2}}^{n_{21}} t_{c_{1}}^{1-n_{11}}\left(n_{21}=+1\right)$

6. Equivalence classes of genus two 3-manifolds and equivalence classes of 6-plat presentations of knots and links. In this section we prove the theorem:

THEOREM 7. Let $Y=Y_{g}$ be a solid handlebody of genus $g$, which is imbedded in $E^{3}$ in the manner indicated in Figure 4. Let $T: Y \rightarrow Y$ be the involution which is induced by rotation about the $z$ axis in Figure 4. Let $\alpha$ : $Y \rightarrow Y$ be a homeomorphism which has the property that $\alpha \mid \partial Y$ commutes with $T \mid \partial Y$. Then there is a homeomorphism $\beta: Y \rightarrow Y$ such that $\beta|\partial Y=\alpha| \partial Y$, and $\beta$ commutes with the involution $T$.

We then use this theorem to show that the natural map from equivalence classes of 6-plat presentations of knots and links to equivalence classes of Heegaard splittings of genus two 3-manifolds is injective and surjective.

In what follows, let $b$ denote the set $Y \cap z$ axis, which is the branch cover of the natural projection $p: Y \rightarrow Y / \tau$. The symbol $D$ denotes the 3-ball $Y / \tau$, and $a=p(b)$ is the branch set.

LEMMA 9. Let $f$ be a proper map of $D^{2}$ into $Y$, such that $f\left(\partial D^{2}\right)$ is a simple closed curve in $\partial Y$ which is disjoint from $T f\left(\partial D^{2}\right)$. There is a proper map $g$ of $D$ into $Y$ such that $g\left|\partial D^{2}=f\right| \partial D^{2}, g$ is an embedding, and $g\left(D^{2}\right) \cap T g\left(D^{2}\right)$ $=\varnothing$. 
Proof of Lemma 9. The idea of the proof is to modify $f$ so that $f\left(D^{2}\right)$ no longer intersects $b$, then project $f$ to obtain a Dehn disc in $D^{3}-a$ and use the Dehn lemma.

Assume $f\left(D^{2}\right)$ intersects $b n$ times. We begin by finding a homotopy, $f \simeq f_{4}$, fixed on $\partial D^{2}$, such that $f_{4}$ intersects $b$-times, and $f_{4}\left(D^{2}\right) \cap T f_{4}\left(D^{2}\right)$ is a 1-complex in which each vertex is the boundary of exactly two or four 1 simplices. We do this via a sequence of homotopies, each fixed on $\partial D^{2}$. Each subsequent function in the following list will be assumed to have all the properties listed for the functions preceding it.

0 . The function $f_{0}$ intersects $b$ exactly $n$ times and is simplicial with respect to some triangulation of $D^{2}$.

1. The function $f_{1} \simeq f_{0}$ is nondegenerate, also $f_{1}$ (1-skeleton) $\cap b=\varnothing$.

2. The function $f_{2} \simeq f_{1}$. Also, if $\sigma_{1}^{2}$ and $\sigma_{2}^{2}$ are 2-simplices such that $f_{2}\left(\sigma_{1}^{2}\right) \cap b \neq \varnothing$ and $f_{2}\left(\sigma_{2}^{2}\right) \cap \neq \varnothing$ then $f_{2}\left(\sigma_{1}^{2}\right) \cap f_{2}\left(\sigma_{2}^{2}\right)=\varnothing$ (we may have to pass to a finer triangulation). Also, if $\sigma_{2}$ is a 2-simplex such that $f_{2}\left(\sigma_{2}\right) \cap b=$ $\varnothing$, then $f_{2}\left(\sigma_{2}\right) \cap \tau f_{2}\left(\sigma_{2}\right)=\varnothing$.

3. The function $f_{3} \simeq f_{2}$. If $f_{3}\left(\sigma^{2}\right) \cap b \neq \varnothing$, then $f_{3}\left(\sigma^{2}\right)$ is not perpendicular to $b$.

4. The function $f_{4} \simeq f_{3}$, also $p f_{4}\left(D^{2}\right)$ is a normal Dehn disc in $D^{3}$. This means that singularities of $p f_{4}\left(D^{2}\right)$ do not occur on the boundary, and are either double points, along which two sheets cross, or triple points at which three sheets cut, or branch points. $\left(Q \in p f_{4}\left(D^{2}\right)\right.$ is a branch point if a small sphere centered at $Q$ cuts $p f_{4}\left(D^{2}\right)$ in a single nonsimple curve.) This may be accomplished by a small homotopy of $p f_{3}$ fixed on $\partial D^{2} \cup$ neighborhood of $f_{3}^{-1}(b)$, which may be lifted to a homotopy of $f_{3}$. We need not homotopy $f_{3}$ at the points of $f_{3}^{-1}(b)$ because by 1,2 and 3 , all the singularities of $p f_{3}\left(D^{2}\right)$ in a neighborhood of $a$ are of the right type.

By 2 and 3 each point $P$ of $f_{4}\left(D^{2}\right) \cap T f_{4}\left(D^{2}\right) \cap b$ has a neighborhood $N$ such that $f_{4}\left(D^{2}\right) \cap T f_{4}\left(D^{2}\right) \cap N$ is a line segment with $P$ in its interior.

If $Q \in f_{4}\left(D^{2}\right) \cap T f_{4}\left(D^{2}\right) \cap(Y-b)$, let $N$ be a spherical neighborhood of $Q$ such that $N \cap T N=\varnothing$. The map $p:\left(f_{4}\left(D^{2}\right) \cup T f_{4}\left(D^{2}\right)\right) \cap N \rightarrow p f_{4}\left(D^{2}\right) \cap$ $p N$ is a homeomorphism. Since $p$ of any point of $f_{4}\left(D^{2}\right) \cap T f_{4}\left(D^{2}\right) \cap N$ is a singular point of $p f_{4}\left(D^{2}\right) \cap N$ we see that $f_{4}\left(D^{2}\right) \cap T f_{4}\left(D^{2}\right)$ is a 1-complex. If $p(Q)$ is a double point then it is the boundary of exactly two one-simplexes in $f_{4}\left(D^{2}\right) \cap T f_{4}\left(D^{2}\right)$. If $p(Q)$ is a triple point then it is the boundary of exactly four one-simplexes in $f_{4}\left(D^{2}\right) \cap T f_{4}\left(D^{2}\right)$. Now $p(Q)$ cannot be a branch point since if that were so the inverse image of the intersection of $p f_{4}\left(D^{2}\right)$ with a small sphere centered at $p(Q)$ would be a simple closed curve in either $f_{4}(N)$ or $T f_{4}(N)$. Since $f_{4}(N) \cap T f_{4}(N)=\varnothing, Q$ could not belong to $f_{4}\left(D^{2}\right) \cap T f_{4}\left(D^{2}\right)$. Thus $f_{4}(D)$ intersects $b m$ times, $f_{4}\left(D^{2}\right) \cap T f_{4}\left(D^{2}\right)$ is a 1-complex in which each 
vertex is the boundary of either two or four 1-simplices.

Now we show that each component of $f_{4}\left(D^{2}\right) \cap T f_{4}\left(D^{2}\right)$ contains an even number of points of $b$. Let $K$ be a component of $f_{4}\left(D^{2}\right) \cap T f_{4}\left(D^{2}\right)$ that contains a point in $b$. Since $f_{4}\left(D^{2}\right) \cap T f_{4}\left(D^{2}\right)$ is invariant under $T$, and the point in $b$ is left fixed by $T$, an easy connectedness argument shows $K$ is invariant under $T$. Triangulate $K$ so the map $p: K \rightarrow K / T$ is simplicial. There are an even number of 1 -simplices in $K$ for this triangulation. We remove the midpoint of each 1 simplex and all the vertices. Note that the number of components is divisible by four, and the closure of each component contains exactly one vertex. Now count the number of components. This number equals $2 \times$ (\# vertices in $K \cap b)+$ $4 \times$ (\# vertices in $p(K)-a$ that are the boundaries of two 1 -simplices) $+8 \times$ (\# vertices in $p(K)$ that are the boundaries of four 1-simplices). Thus the number of vertices in $K \cap b$ is even. Now we show how to modify $f_{4}$ so as to eliminate two intersections of $f_{4}(D)$ with $b$. Let $K$ be a component of $f_{4}\left(D^{2}\right) \cap T f_{4}\left(D^{2}\right)$ containing points of $b$ and let $\delta$ be a simple arc in $p(K)$ containing two distinct points of $a$ for endpoints and no points of $a$ in its interior. $p^{-1}(\delta)$ is a simple closed curve in $Y$ and $f_{4}^{-1} p^{-1}(\delta)$ contains a simple closed curve in $D^{2}$ bounding a disc we shall call $E$. We may choose a parametrization, $\gamma(s), 0 \leqslant s \leqslant 1$, of $f_{4}^{-1} p^{-1}(\delta)$ such that $f_{4}(\gamma(s))=T f_{4}(\gamma(1-s)), 0 \leqslant s \leqslant 1$. Now let $Q$ be a homeomorphism of the disc $E$ such that $Q(\gamma(s))=\gamma(1-s)$. Define the function $f_{5}: f_{5}(x)=f_{4}(x)$ if $x \notin E$, and $f_{5}(x)=T f_{4}(Q(x))$ if $x \in E$. Note that $f_{5}$ is well defined since $f_{5}(\gamma(s))=T f_{4}(Q(\gamma(s)))=T f_{4}(\gamma(1-s))=f_{4}(\gamma(s))$. We have not altered the fact that $f_{5}(D) \cap b$ is $n$ points. Now examine $f_{4}$ and $f_{5}$ in a neighborhood of $\gamma(0)$. Note that $\gamma(0)$ belongs to the interior of a simplex $\sigma^{2}$ which $f_{4}$ linearly embeds in $Y$, in such a way that $f_{4}\left(\sigma^{2}\right)$ is not perpendicular to $b$. Thus there is a unique line segment in $f_{4}\left(\sigma^{2}\right)$ that is perpendicular to $b$. This line segment and $b$ define a plane $P$, which divides $E^{3}$ into two closed half-spaces $H_{1}$ and $H_{2}$. Examining the definition of $f_{5}$ we see that there is a neighborhood $N$ of $\gamma(0)$ such that $f_{5}(N)$ lies entirely in one of these half-spaces. Now it is easy to find a homotopy $f_{6} \simeq f_{5}$ fixed on the complement of the interior of $N$ such that $f_{6}(N) \cap b=\varnothing$. In a similar manner we may find a neighborhood $N^{\prime}$ of $\gamma(1 / 2)$ and a homotopy $f_{7} \simeq f_{6}$ fixed on the complement of the interior of $N^{\prime}$ such that $f_{7}\left(N^{\prime}\right) \cap b=\varnothing$. In summary we have found a function $f_{7}$ such that $f_{7}\left|\partial D^{2}=f\right| \partial D^{2}, f_{7}\left(D^{2}\right) \cap b$ contains two fewer points then $f\left(D^{2}\right) \cap b$, and if $f_{7}\left(\sigma^{2}\right) \cap b \neq \varnothing$ then $f_{7}\left(\sigma^{2}\right) \cap b$ belongs to the interior of $f_{7}\left(\sigma^{2}\right)$. Repeating the same process $n / 2$ times we finally arrive at a function $g_{1}$ such that $g_{1}\left(D^{2}\right) \cap$ $b=\varnothing$ and $g_{1}\left|\partial D^{2}=f\right| \partial D^{2}$.

Since $g_{1}\left(\partial D^{2}\right)$ is a simple closed curve in $\partial Y$ disjoint from $T g_{1}\left(\partial D^{2}\right)$, it follows that $p g_{1}\left(D^{2}\right)$ is a singular Dehn disc in $D^{3}-a$. By the Dehn lemma [26] there is a function $\tilde{g}$ such that $\tilde{g}\left(D^{2}\right)$ is a nonsingular disc in $D^{3}-a$ and 
$\tilde{g}\left|\partial D^{2}=g_{1}\right| \partial D^{2}$. We may lift $\tilde{g}$ to a function $g: D^{2} \rightarrow Y$ such that $g$ satisfies the conclusion of Lemma 9.

Proof of Theorem 7. Now note that $\left(D^{3}, a\right)$ is a 3-ball with $g+1$ distinguished disjoint unknotted, unlinked, and proper arcs, where $g$ is the genus of $Y$. Since $\alpha \mid \partial Y$ commutes with $T$ it projects to a homeomorphism $\underline{\alpha}$ of the 2 sphere $\partial D^{3}$ that takes $\partial a$ into itself. Let $\underline{D}_{1}^{2}, \ldots, \underline{D}_{g}^{2}$ be a set of disjoint proper discs in $D^{3}-a$ such that $D^{3}-\bigcup_{i=1}^{g} \underline{D}_{i}^{2}$ consists of $g+1$ components each of which contains one distinguished arc. Let $D_{i}^{2}$ be one of the two lifts of $\underline{D}_{i}^{2}$ to $Y$. This function $\alpha$ restricted to $D_{i}^{2}$ satisfies the hypothesis of the preliminary lemma. Hence there is a disc $E_{i}^{2}$ such that $\alpha\left(\partial D_{i}^{2}\right)=\partial E_{i}^{2}$ and $E_{i}^{2} \cap T E_{i}^{2}=\varnothing$. Let $\underline{E}_{i}^{2}=$ $p\left(E_{i}^{2}\right)$. In general the set $\left\{E_{i}^{2}\right\}$ will not be pairwise disjoint. However, we may systematically eliminate intersections and replace the $\left\{\underline{E}_{i}^{2}\right\}$ with a set of pairwise disjoint proper discs $\left\{\underline{F}_{i}^{2}\right\}$ such that $\underline{F}_{i}^{2}=\underline{\alpha}\left(\partial \underline{D}_{i}^{2}\right)$. To see how to do that suppose $\underline{E}_{i}^{2}$ and $\underline{E}_{j}^{2}$ intersect. We may suppose they are in general position and so they intersect in a set of circles. Let $c$ be a circle of intersection such that $c$ bounds a disc $G^{2}$ in $\underline{E}_{i}^{2}$ not containing any other intersection points in $\underline{E}_{i}^{2}$ and $c$ bounds a disc $H^{2}$ in $E_{j}^{2}$. Since $H^{2}$ and $G^{2}$ do not intersect they bound a ball $J^{3}$ in $D^{3}-a$. There is an isotopy $\varphi$ supported on a regular neighborhood of the ball $J^{3}$ such that $\varphi$ "pushes" $G^{2}$ through $H^{2}$. If we replace the disc $E_{i}^{2}$ with $\varphi\left(E_{i}^{2}\right)$ we reduce the number of circles of intersection by at least one. We can continue to do this, being careful at each stage not to introduce new intersections, until all the intersections are eliminated. Now extend $\underline{\alpha}$ to $\partial D^{3} \cup \bigcup_{i=1}^{g} \underline{D}_{i}^{2}$ by letting $\underline{\alpha}$ map $\underline{D}_{i}^{2}$ homeomorphically onto $\underline{F}_{i}^{2}$. Since $\partial \underline{D}_{i}^{2}$ divided $\partial D^{3}$ into $g+1$ regions $R_{0}^{2}, \ldots, R_{g}^{2}$ each containing two points of $\partial a$, the regions $\underline{\alpha}\left(R_{0}^{2}\right), \ldots, \underline{\alpha}\left(R_{g}^{2}\right)$ also each contain two points of $\partial a$. Thus we have $g+1$ regions $S_{0}^{3}, \ldots, S_{g}^{3}$ each of which is a ball containing one arc of $a$ and another $g+1$ regions $T_{0}^{3}$, $\ldots, T_{g}^{3}$, each of which is a ball containing one arc of $a$. We also have a homeomorphism $\underline{\alpha}: \partial S_{i}^{3} \rightarrow \partial T_{i}^{3}$ that preserves the set $\partial a$. Now we extend $\underline{\alpha}$ in any way at all to a homeomorphism $\underline{\alpha}: S_{i} \rightarrow T_{i}$ and, making use of the fact that any two unknotted arcs in a ball are isotopic, find an isotopy $\psi$ such that $\psi \alpha$ is a homeomorphism sending $a$ into $a$ and $\underline{\psi \alpha} \mid \partial D^{3}=\underline{\alpha}$. Now we may lift $\underline{\psi} \underline{\alpha}$ to get the desired homeomorphism $\beta$ of $Y$. This completes the proof of Theorem 7 .

We recall from $\$ 4$ that a $2 b$ plat is a representation of a knot or link of form $\left(D, A_{b}\right) \cup_{\tau \delta}\left(D^{\prime}, A_{b}^{\prime}\right)$ where $D$ is a 3-ball containing $A_{b}$, a set of $b$ unlinked unknotted arcs, $\left(D^{\prime}, A_{b}^{\prime}\right)$ is another copy of $\left(D, A_{b}\right), \tau$ is the map identifying $D$ with $D^{\prime}$, restricted to $\partial D$, and $\delta$ is a homeomorphism of $\partial D$ leaving the points in $\partial A_{b}$ invariant as a set.

We say the plat $\left(D, A_{b}\right) \cup_{\tau \delta}\left(D^{\prime}, A_{b}^{\prime}\right)$ is equivalent to the plat $\left(D, A_{b}\right) \cup_{\tau \psi}$ $\left(D^{\prime}, A_{b}^{\prime}\right)$ if there are orientation-preserving homeomorphisms $\alpha, \alpha^{\prime}$ of $\left(D, A_{b}\right)$ and $\left(D^{\prime}, A_{b}^{\prime}\right)$ respectively, such that $\left(\alpha^{\prime} \mid \partial D^{\prime}\right) \tau \delta=\tau \psi(\alpha \mid \partial D)$. 
Recall from $\S 4$ that $Y_{g} \cup_{\tau \mu} Y_{g}^{\prime}$ defines a Heegaard splitting of a 3-manifold; $\pi:\left(Y_{g}, L_{b}\right) \rightarrow\left(D, A_{b}\right)$ and $\pi^{\prime}:\left(Y_{g}^{\prime}, L_{g}^{\prime}\right) \rightarrow\left(D^{\prime}, A_{b}^{\prime}\right)$ are $b$-fold branched cyclic coverings, with branch covers the sets $L_{b}$ and $L_{b}^{\prime}$ respectively. We say the Heegaard splittings $Y_{g} \cup_{\tau \mu} Y_{g}^{\prime}$ and $Y_{g} \cup_{\tau^{\prime} \nu} Y_{g}^{\prime}$ are equivalent if there are orientation preserving homeomorphisms $\tilde{\alpha}$ and $\tilde{\alpha}^{\prime}$ of $Y_{g}$ and $Y_{g}^{\prime}$ respectively such that $\left(\tilde{\alpha}^{\prime} \mid \partial Y_{g}^{\prime}\right) \tau \mu$ $=\tau \nu\left(\widetilde{\alpha} \mid \partial Y_{g}\right)$.

Let $F_{b, g}$ be the map that assigns to an equivalence class of plats $\left\{\left(D, A_{b}\right)\right.$ $\left.\cup_{\tau \delta}\left(D^{\prime}, A_{b}^{\prime}\right)\right\}$ the equivalence class of Heegaard splittings $\left\{Y_{g} \cup_{\tau \mu} Y_{g}^{\prime}\right\}$ where $\mu$ is the lift to $\partial Y_{g}$ of the homeomorphism $\delta$ of $\partial D . F_{b, g}$ is well defined because homeomorphisms $\alpha, \alpha^{\prime}$ of $\left(D, A_{b}\right)$ and $\left(D^{\prime}, A_{b}^{\prime}\right)$ lift to homeomorphisms $\tilde{\alpha}, \tilde{\alpha}^{\prime}$ of $Y_{g}, Y_{g}^{\prime}$ and the commutative diagram defining equivalence downstairs lifts to a commutative diagram defining equivalence upstairs. At this point, we specialize to the case $b=3, g=2$ and denote $F_{3,2}$ simply by $F$. We wish to show that $F$ is injective and surjective. (For $g \geqslant 3, F_{b, g}$ is probably not surjective as there are isotopy classes of homeomorphisms of $\partial Y_{g}$ that have no representative commuting with the covering transformation.)

THEOREM 8. The natural map $F$ from equivalence classes of 6-plats to equivalence classes of genus 2 Heegaard splittings is injective and surjective.

Proof of TheOREM 8. Surjectivity follows easily from the fact that any homeomorphism of $\partial Y_{2}$ is isotopic to one commuting with the covering transformation in the covering $\pi: \partial Y_{2} \rightarrow \partial D$.

Suppose $Y_{2} \cup_{\tau \mu} Y_{2}^{\prime} \equiv Y_{2} \cup_{\tau \nu} Y_{2}^{\prime}$. Then there are orientation-preserving homeomorphisms $\tilde{\alpha}, \tilde{\alpha}_{\alpha}$ of $Y_{2}$ and $Y_{2}^{\prime}$ such that $\left(\tilde{\alpha}^{\prime} \mid \partial Y_{2}^{\prime}\right) \tau \mu=\tau \nu\left(\tilde{\alpha} \mid \partial Y_{2}\right)$. Since the genus is $2, \tilde{\alpha} \mid \partial Y_{2}$ is isotopic to a homeomorphism $\tilde{\alpha}_{1}$ of $\partial Y_{2}$ where $\tilde{\alpha}_{1}$ commutes with the covering transformation. We can extend the isotopy to all of $Y_{2}$. Call the isotopy $\tilde{\alpha}_{t}$. Then we can use $\tilde{\alpha}_{t}$ to define an isotopy of $\widetilde{\alpha}^{\prime} \mid \partial Y_{2}^{\prime}$, defining $\tilde{\alpha}_{t} \mid \partial Y_{2}^{\prime}$ by the equation $\left(\tilde{\alpha}_{t}^{\prime} \mid \partial Y_{2}^{\prime}\right) \tau \mu=\tau \nu\left(\tilde{\alpha}_{t} \mid \partial Y_{2}\right)$. This is possible since $\mu, \nu, \tau$ and $\tilde{\alpha}_{t}$ are all homeomorphisms. Thus $\left(\tilde{\alpha}_{1}^{\prime} \mid \partial Y_{2}^{\prime}\right) \tau \mu=\tau v\left(\tilde{\alpha}_{1} \mid \partial Y_{2}\right)$. Now $\tilde{\alpha}_{1}^{\prime}$ must be fibre preserving since $\tilde{\alpha}_{1}, \mu, \nu$ and $\tau$ are all fibre preserving. Thus $\tilde{\alpha}_{1}$ commutes with the covering transformation. Since the isotopy $\tilde{\alpha}_{t}^{\prime}$ may be extended to an isotopy of $Y_{2}^{\prime}$ we could use the homeomorphisms $\widetilde{\alpha}_{1}$ and $\widetilde{\alpha}_{1}^{\prime}$ in place of the homeomorphisms $\tilde{\alpha}$ and $\tilde{\alpha}^{\prime}$ to define the equivalence of Heegaard splittings. Thus we may assume that $\tilde{\alpha} \mid \partial Y_{2}$ and $\tilde{\alpha}^{\prime} \mid \partial Y_{2}^{\prime}$ commute with the covering transformation. By Theorem 7 we may choose extensions of $\tilde{\alpha} \mid \partial Y_{2}$ and $\tilde{\alpha}^{\prime} \mid \partial Y_{2}^{\prime}$ that commute with the covering transformation in the coverings $\pi: Y_{2} \rightarrow D$ and $\pi^{\prime}: Y_{2}^{\prime} \rightarrow D^{\prime}$. Thus we may assume $\tilde{\alpha}$ and $\tilde{\alpha}^{\prime}$ had this property to begin with. Now $\tilde{\alpha}$, and $\tilde{\alpha}^{\prime}$ project to homeomorphisms $\alpha$ and $\alpha^{\prime}$ with $\left(\alpha^{\prime} \mid \partial D^{\prime}\right) \tau \delta=\tau \psi(\alpha \mid \partial D)$ so that $\left(D, A_{3}\right) \cup_{\tau \delta}\left(D^{\prime}, A_{3}^{\prime}\right) \equiv\left(D, A_{3}\right) \cup_{\tau \psi}\left(D^{\prime}, A_{3}^{\prime}\right)$.

Theorem 8 sheds light on a question raised by Papakyriakopoulos [28, 
p. 330]. He asks whether any two Heegaard splittings of a 3-manifold are equivalent. $\left({ }^{9}\right.$ ) (His definition of equivalence is somewhat different from ours in that he allows homeomorphisms of $M=Y_{g} \cup_{\tau \mu} Y_{g}^{\prime}$ onto $M=Y_{g} \cup_{\tau \nu} Y_{g}^{\prime}$ that map $Y_{g}$ onto $Y_{g}^{\prime}$. We could have used a similar definition of equivalence (suitably modifying our definition of equivalence of plats) and obtained a theorem similar to Theorem 8 , but the exposition would have been more complicated.) Theorem 8 shows that, for genus 2 , the existence of two nonequivalent 6-plat presentations of a prime knot or link would imply a negative answer to Papakyriakopoulos' question.

Theorem 8 and the results of $\S 4$ indicate that the following question is interesting, and may be easier to answer than the analogous question about 3 manifolds: Are any two "Heegaard splittings" of a prime knot equivalent?* i.e. let $D \cup_{\tau \psi} D^{\prime}$ be a Heegaard splitting of $S^{3}$. Let $A_{b}$ be a collection of $b$ unknotted and unlinked arcs in $D$ with endpoints on $\partial D$, and let $A_{b}^{\prime}=\tau\left(A_{b}\right)$. Then $L_{\psi}=$ $A_{b} \cup_{\tau \psi} A_{b}^{\prime}$ is a link in $S^{3}=D \cup_{\tau \psi} D^{\prime}$, represented as a $2 b$-plat. $\left({ }^{10}\right)$ Let $B_{2 b}$ be the group of all autohomeomorphisms of $\left(\partial D, A_{b}\right)$, and let $G$ be the subgroup of those autohomeomorphisms which extend to $\left(D, A_{b}\right)$.

Question. Suppose that $L$ is prime. Are all $2 b$-plat representatives of the link type of $L_{\psi}$ obtainable as $A_{b} \cup_{\tau \varphi} A_{b}^{\prime}$, where $\varphi=\psi_{1} \cdot \psi \cdot \psi_{2}$, with $\psi_{1}, \psi_{2}$ $\in G$ ?

7. Presentations for $\pi_{1} S^{3}$. If a 3-manifold $M$ is defined by a Heegaard splitting $Y_{g} \cup_{\tau \Phi} Y_{g}^{\prime}$, and if the action of the defining homeomorphism on $\pi_{1}\left(\partial Y_{g}\right)$ is known, then there is a very easy algorithm which allows us to determine a presentation for $\pi_{1}(M)$. This algorithm will be presented in Lemma 10. We will then study the question: If $M \sim S^{3}$, what types of "canonical" presentations can we expect for $\pi_{1}(M)=1$ ? To attack this question, we first characterize algebraically the class of elements in the mapping class group of the surface $\partial Y_{g}$ which have the property that they define Heegaard splittings of $S^{3}$ (Lemma 11). It will then be established in Theorem 9 that $\pi_{1} S^{3}$ can, in fact, have very "bad" presentations which arise in a natural manner in connection with Heegaard splittings.

We will end this section with an observation which relates in particular to 2-symmetric Heegaard splittings: If $M=Y_{g} \cup_{\tau \Phi} Y_{g}^{\prime}$, is 2-symmetric, then $\pi_{1} M$

(9) For composite manifolds the question was answered in the negative by Engmann, Nicht-homöomorphe Heegaard-Zerlegungen vom Geschlect 2 der zusammenhängenden Summe zweier Linsenrdume, Abh. Math. Sem. Univ. Hamburg 35 (1971), 33-38.

*Note added in proof. This question has been answered in the negative. Theorem 8 was used by Birman, Gonzalez-Acuña, and Montesinos to exhibit two inequivalent Heegaard splittings of a prime genus two $Z$ homology sphere, Heegaard splittings of prime 3-manifolds are not unique (to appear).

(10) The autohomeomorphism $\psi$ defines the $2 b$-braid which determines our plat, as in equations (6) and (7) of $\S 5$. 
has a "symmetric" presentation (Theorem 10).

To determine a presentation for the fundamental group of a 3-manifold which is defined by a Heegaard splitting $Y_{g} \cup_{\tau \Phi} Y_{g}^{\prime}$, we first choose canonical generators for $\pi_{1}\left(\partial Y_{g}\right)$. These generators will be denoted by $w_{1}, \ldots, w_{2 g}$ and will be assumed to satisfy the single defining relation

$$
\prod_{k=1}^{g} w_{k} w_{g+k} w_{k}^{-1} w_{g+k}^{-1}=1
$$

Let $\vec{u}=\left\{u_{1}, \ldots, u_{g}\right\}$ be a free basis for $\pi_{1} Y_{g}$, where if $i: \partial Y_{g} \rightarrow Y_{g}$ denotes the inclusion map, and $i_{*}: \pi_{1} \partial Y_{g} \rightarrow \pi_{1} Y_{g}$ denotes the homomorphism induced by $i$, then

$$
\begin{array}{lr}
i_{*}: w_{j} \rightarrow u_{j}, & 1 \leqslant j \leqslant g, \\
i_{*}: w_{j} \rightarrow 1, & g+1 \leqslant j \leqslant 2 g .
\end{array}
$$

Let $w_{j}^{\prime}, u_{j}^{\prime}, i^{\prime}, i_{*}^{\prime}, \vec{u}$ be defined in an analogous manner for the handlebody $Y_{g}^{\prime}$. Suppose also that the homeomorphism $\tau: Y_{g} \rightarrow Y_{g}^{\prime}$ has the effect:

$$
\tau_{*}: w_{j} \rightarrow w_{j}^{\prime}, \quad 1 \leqslant j \leqslant 2 g .
$$

The defining homomorphism $\Phi: \partial Y_{g} \rightarrow \partial Y_{g}$ will be assumed to induce an automorphism $\Phi_{*}: \pi_{1} \partial Y_{g} \rightarrow \pi_{1} \partial Y_{g}$ which acts as follows:

$$
\begin{aligned}
\Phi_{*}: w_{j} & \rightarrow U_{j}\left(w_{1}, \ldots, w_{2 g}\right), \\
\Phi_{*}^{-1}: w_{j} & \rightarrow U_{j}^{\prime}\left(w_{1}, \ldots, w_{2 g}\right), \quad 1 \leqslant j \leqslant 2 g .
\end{aligned}
$$

Then we claim

LEMMA 10. $\pi_{1}\left(Y_{g} \cup_{\tau \Phi} Y_{g}^{\prime}\right)$ admits the following two presentations:

(i) $\left\langle u_{1}^{\prime}, \ldots, u_{g}^{\prime} ; U_{k}\left(u_{1}^{\prime}, \ldots, u_{g}^{\prime}, 1, \ldots, 1\right), g+1 \leqslant k \leqslant 2 g\right\rangle$.

(ii) $\left\langle u_{1}, \ldots, u_{g} ; U_{k}^{\prime}\left(u_{1}, \ldots, u_{g}, 1, \ldots, 1\right), g+1 \leqslant k \leqslant 2 g\right\rangle$.

Proof of Lemma 10. The presentations of Lemma 10 are obtained by a straightforward application of the van Kampen theorem.

The presentation (i) (or (ii)) of Lemma 10 defines a homomorphism from the free group $F_{g}$ of rank $g$ with free basis $\vec{u}$ (or $\vec{u}$ ) onto $\pi_{1}\left(Y_{g} \cup_{\tau \Phi} Y_{g}^{\prime}\right.$ ). A natural question to ask, if one is attempting to understand the relationship between the algebraic and geometric properties of 3-manifolds, is whether the trivial group always appears in these particular "canonical" presentations in a "nice" way. For example, an obvious first guess would be that perhaps $\pi_{1}\left(Y_{g} \cup_{\tau \Phi} Y_{g}^{\prime}\right)=1$ only if the set $\vec{U}=\left\{U_{k}\left(u_{1}^{\prime}, \ldots, u_{g}^{\prime}, 1, \ldots, 1\right)\right\}$ are a set of primitive elements in the free group $F_{g}$. This is false, as will be seen below. (This appears to be a known result, although we were unable to locate an explicit reference in the published literature.) More subtly, it has been conjectured by W. Haken that if $Y_{g} \cup_{\tau \Phi} Y_{g}^{\prime}$ 
is $S^{3}$, then at least one of the presentations (i) or (ii) of Lemma 10 has the property that the relators are a set of conjugate primitive elements. This conjecture is, unfortunately, laid to rest below:

THEOREM 9. There exists a genus 2 Heegaard splitting of $S^{3}$ which has the property that neither of the sets $\left\{U_{3}\left(u_{1}^{\prime}, u_{2}^{\prime}, 1,1\right), U_{4}\left(u_{1}^{\prime}, u_{2}^{\prime}, 1,1\right)\right\}$ or $\left\{U_{3}^{\prime}\left(u_{1}, u_{2}, 1,1\right), U_{4}^{\prime}\left(u_{1}, u_{2}, 1,1\right)\right\}$ is a conjugate primitive set.

Proof. Our proof begins with the establishment of several lemmas, the first of which may have some interest in its own right.

Lemma 11. Let $Y \cup_{\tau \Phi_{0}} Y_{g}^{\prime}$ be any "standard" Heegaard splitting of $S^{3}$, and let $Y_{g} \cup_{\tau \Phi} Y_{g}^{\prime}$ be an arbitrary Heegaard splitting. Then $Y_{g} \cup_{\tau \Phi} Y_{g}^{\prime}$ is $S^{3}$ if and only if

$$
\Phi=\Phi_{1} \Phi_{0} \Phi_{2},
$$

when $\Phi_{1}$ and $\Phi_{2}$ are surface homeomorphisms which extend to homeomorphisms of $Y_{g}$.

Proof of Lemma 11. Lemma 11 will be shown to follow from results of Waldhausen in [33]. It was established by Waldhausen that every "minimal" Heegaard splitting of $S^{3}$ has genus 0 . (The reader is referred to [33] for the definition of a minimal Heegaard splitting.) An induction based on genus then implies that if $Y_{g} \cup_{\tau \Phi} Y_{g}^{\prime} \sim S^{3}$, then there exists a homeomorphism

$$
h: Y_{g} \cup_{\tau \Phi} Y_{g}^{\prime} \rightarrow Y_{g} \cup_{\tau \Phi_{0}} Y_{g}^{\prime}
$$

which maps $Y_{g} \rightarrow Y_{g}, Y_{g}^{\prime} \rightarrow Y_{g}^{\prime}$, and is consistent on the boundary, that is, the diagram

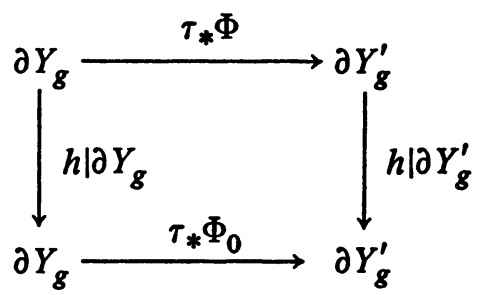

is commutative. Thus

$$
\Phi=\tau_{*}^{-1}\left(h \mid \partial Y_{g}^{\prime}\right) \tau_{*} \Phi_{0}\left(h \mid \partial Y_{g}\right)
$$

Setting $\Phi_{1}=\tau_{*}^{-1}\left(h \mid \partial Y_{g}^{\prime}\right) \tau_{*}$ and $\Phi_{2}=h \mid \partial Y_{g}$, we then obtain the desired result. To establish sufficiency, suppose that $M=Y_{g} \cup_{\tau \Phi} Y_{g}^{\prime}$, where $\Phi$ satisfies condition (18) of Lemma 11. Let $h_{1}, h_{2}$ be extensions of $\Phi_{1}, \Phi_{2}$ respectively to $Y_{g}$. We may then define a homeomorphism $h: Y_{g} \cup_{\tau \Phi} Y_{g}^{\prime} \rightarrow Y_{g} \cup_{\tau \Phi_{0}} Y_{g}^{\prime}$ by the rule $h \mid Y_{g}=h_{2}$ and $h \mid Y_{g}^{\prime}=\tau^{-1} h_{1} \tau$. Condition (18) ensures that $h$ is well 
defined on $\partial Y_{g}$. Since $Y_{g} \cup_{\tau \Phi_{0}} Y_{g}^{\prime} \sim S^{3}$, it then follows that $Y_{g} \cup_{\tau \Phi} Y_{g}^{\prime}$ is also $S^{3}$. This proves Lemma 11 .

Our next two lemmas are very easy:

Lemma 12. Let $\vec{U}$ be a set of $g$ elements of $F_{g}$. Let $\beta \in$ Aut $F_{g}$. Then $\beta(\vec{U})$ is a conjugate primitive set only if $\vec{U}$ is a conjugate primitive set.

Proof of Lemma 12. This follows immediately from the definition of a primitive set and a conjugate primitive set.

LEMMA 13. Let $\Phi_{1}: \partial Y_{g} \rightarrow \partial Y_{g}$ be a homeomorphism which extends to $Y_{g}$ Suppose that the action of $\Phi_{1}$, is given by

$$
\Phi_{1}: w_{j} \rightarrow R_{j}\left(w_{1}, \ldots, w_{2 g}\right), \quad 1 \leqslant j \leqslant 2 g .
$$

Then

$$
\dot{R_{j}}\left(u_{1}, \ldots, u_{g}, 1, \ldots, 1\right)=1 \text { for each } g+1 \leqslant j \leqslant 2 g
$$

and

$$
\left\{R_{j}\left(u_{1}, \ldots, u_{g}, 1, \ldots, 1\right), 1 \leqslant j \leqslant g\right\}
$$

are a basis for the free group with free basis $u_{1}, \ldots, u_{g}$.

Proof of Lemma 13. Let $i_{*}: \pi_{1}\left(\partial Y_{g}\right) \rightarrow \pi_{1} Y_{g}$ be the homomorphism induced by the inclusion map. A necessary and sufficient condition for $\Phi_{1}$ to extend to $X_{g}$ is that $\Phi_{1}$, leave ker $i_{*}$ invariant [22], and since ker $i_{*}$ is the normal closure of $\left\{w_{g+1}, \ldots, w_{g}\right\}$ in $\pi_{1}\left(\partial Y_{g}\right)$, it follows that (19) must be true, and also $\Phi_{1}$ induces an automorphism $\Phi_{1 * *}: \pi_{1} Y_{g} \rightarrow \pi_{1} Y_{g}$, defined by

$$
\Phi_{1 \ldots *}: u_{j} \rightarrow R_{j}\left(u_{1}, \ldots, u_{g}, 1, \ldots, 1\right), \quad 1 \leqslant j \leqslant g .
$$

This proves Lemma 13.

We are now ready to prove Theorem 9 . We begin by producing a genus 2 Heegaard splitting of $S^{3}$ which has the property that, in the presentation (i), neither of the relators is conjugate to a primitive element. Explicitly, $\left.{ }^{(11}\right)$ let

$$
\Phi=t_{4}^{-1} t_{5}^{2} t_{4}^{-2} t_{2}^{-2} t_{1} t_{2}^{-1} t_{1}^{-1} t_{2}^{-1} t_{3}^{-1} t_{4}^{-1} t_{5} t_{4} t_{3} t_{2} t_{1},
$$

where $t_{1}, t_{2}, t_{3}, t_{4}, t_{5}$ are the Lickorish twists about the curves $c_{1}, c_{2}, c_{3}, c_{4}, c_{5}$ defined in Figure 4 (cf. §3). To see that $Y_{2} U_{\tau \Phi} Y_{2}^{\prime}$ is $S^{3}$, we note that the algorithm given in $\S 4$ is applicable, because every genus 2 Heegaard splitting is 2-symmetric. We thus construct the 6-plat corresponding to $\Phi$ (Figure 11). Since the plat defines the trivial knot type, it follows from Theorem 6 that $Y_{2} U_{\tau \Phi} Y_{2}^{\prime}$ $\sim S^{3}$.

(11) This example is a modification of an example which was shown to the authors by C. Miller, who attributes it to Reidemeister. We were unable to find a reference in Reidemeister's works. 


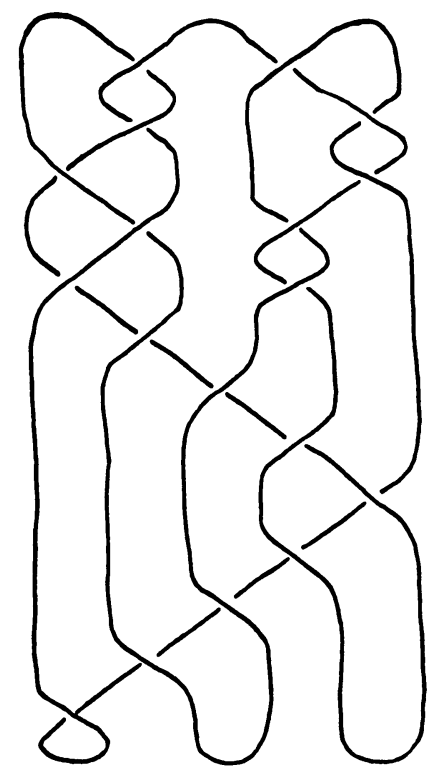

Figure 11. Plat corresponding to the genus 2 Heegaard splitting

$$
\Phi=t_{4}^{-1} t_{5}^{2} t_{4}^{-2} t_{2}^{-2} t_{1} t_{2}^{-1} t_{1}^{-1} t_{2}^{-1} t_{3}^{-1} t_{4}^{-1} t_{5} t_{4} t_{3} t_{2} t_{1}
$$

To compute the action of $\Phi_{*}$ on $\pi_{1}\left(\partial Y_{2}\right)$ we first observe that the twists $t_{1}, \ldots, t_{5}$ induce the following automorphisms of $\pi_{1}\left(\partial Y_{2}\right)$ :

$$
\begin{aligned}
& t_{1}: w_{1} \rightarrow w_{1} w_{3}^{-1}, \quad t_{2}: w_{3} \rightarrow w_{3} w_{1} \text {, } \\
& t_{3}: w_{1} \rightarrow w_{1} w_{3}^{-1} w_{2} w_{4} w_{2}^{-1}, \quad t_{4}: w_{4} \rightarrow w_{4} w_{2} \text {, } \\
& w_{2} \rightarrow w_{2} w_{4}^{-1} w_{2}^{-1} w_{3} w_{2}, \quad t_{5}: w_{2} \rightarrow w_{2} w_{4}^{-1} \text {, } \\
& w_{3} \rightarrow w_{2} w_{4}^{-1} w_{2}^{-1} w_{3} w_{2} w_{4} w_{2}^{-1} \text {, }
\end{aligned}
$$

where every generator which is not listed explicitly is kept fixed. The action of $\Phi_{*}$ on the generators $w_{3}, w_{4}$ of $\pi_{1} \partial Y_{2}$ may now be computed, using equations (22) and (23):

$$
\begin{aligned}
\Phi_{*}: & w_{3} \rightarrow w_{2}^{2} w_{4}^{-1} w_{2} w_{4}^{-1} w_{1} w_{3} w_{1}^{-3} \\
w_{4} & \rightarrow w_{1}^{3} w_{3}^{-1} w_{2} w_{4} w_{2}^{-2} w_{4} w_{2}^{-1} w_{4} w_{2}^{-2}
\end{aligned}
$$

Thus, from Lemma 10, $\pi_{1}\left(Y_{2} \cup_{\tau \Phi} Y_{2}^{\prime}\right)$ admits the presentation

$$
\text { (i) }\left\langle u_{1}^{\prime}, u_{2}^{\prime}: u_{2}^{\prime 3} u_{1}^{\prime-2}, u_{1}^{\prime 3} u_{2}^{\prime-4}\right\rangle \text {. }
$$

One verifies immediately by a standard test (see [20, Corollary N4]) that neither $u_{2}^{\prime 3} u_{1}^{\prime-2}$ nor $u_{1}^{\prime 3} u_{2}^{\prime-4}$ is conjugate to a primitive element in the free group with free basis $u_{1}^{\prime}, u_{2}^{\prime}$. If the presentation (ii) had a similar property, we would be done: however if one does a similar calculation, replacing the automorphism $\Phi_{*}$ by $\Phi_{*}^{-1}$, one discovers that the words $U_{3}^{\prime}\left(u_{1}, u_{2}, 1,1\right)$ and $U_{4}^{\prime}\left(u_{1}, u_{2}, 1,1\right)$ are 
in fact primitive. Hence we need to do some more work.

Let $\Phi_{0}: \partial Y_{2} \rightarrow \partial Y_{2}$ be the twist product

$$
\Phi_{0}=t_{4} t_{5} t_{4} t_{1} t_{2} t_{1} .
$$

Applying the algorithms of $\S 5$, we verify that the 6-plat determined by the 6 braid $\sigma_{4} \sigma_{5} \sigma_{4} \sigma_{1} \sigma_{2} \sigma_{1}$ defines the trivial knot type, hence $Y_{2} \cup_{\tau \Phi_{0}} Y_{2}^{\prime} \sim S^{3}$. Therefore, by Lemma 11, it follows that $\Phi=\Phi_{1} \Phi_{0} \Phi_{2}$, where $\Phi_{1}$ and $\Phi_{2}$ extend to $Y_{2}$. We will now show that if

$$
\Psi=\Phi_{2}^{-1} \Phi_{0} \Phi_{2},
$$

then $Y_{2} \cup_{\tau \Psi} Y_{2}^{\prime}$ is $S^{3}$, and also $\pi_{1}\left(Y_{2} \cup_{\tau \Psi} Y_{2}^{\prime}\right)$ admits two canonical presentations (i) and (ii), as described in Lemma 10 , which have the property that the relators in each of these presentations fail to be a conjugate primitive set. This will provide the example needed to complete the proof of our theorem.

To see that $Y_{2} \cup_{\tau \Psi} Y_{2}^{\prime}$ is $S^{3}$, note that $\Phi_{2}$ extends to $Y_{2}$, hence also $\Phi_{2}^{-1}$ extends to $Y_{2}$, hence by Lemma 11 it must be true that $Y_{2} \cup_{\tau \Psi} Y_{2}^{\prime} \sim S^{3}$.

To see that our canonical presentation has the required properties, suppose that the action of $\Phi_{1}$, and $\Phi_{2}$, on $\pi\left(\partial Y_{2}\right)$ is given by

$$
\begin{aligned}
& \Phi_{1_{*}}: w_{j} \rightarrow R_{j}\left(w_{1}, w_{2}, w_{3}, w_{4}\right) \\
& \Phi_{2_{*}: w_{j}} \rightarrow S_{j}\left(w_{1}, w_{2}, w_{3}, w_{4}\right) \\
& \Phi_{2}^{-1}: w_{j} \rightarrow T_{j}\left(w_{1}, w_{2}, w_{3}, w_{4}\right), \quad 1 \leqslant j \leqslant 4 .
\end{aligned}
$$

Note that the action of $\Phi_{0}$ and $\Phi_{0_{*}}^{-1}$ may be computed from equations (23) and (26) to be

$$
\begin{aligned}
\Phi_{0 *}: w_{1} & \rightarrow w_{3}^{-1}, & \Phi_{0 *}^{-1}: w_{1} & \rightarrow w_{1} w_{2} w_{1}^{-1}, \\
w_{2} & \rightarrow w_{4}^{-1}, & w_{2} & \rightarrow w_{2} w_{4} w_{2}^{-1}, \\
w_{3} & \rightarrow w_{3} w_{1} w_{3}^{-1}, & w_{3} & \rightarrow w_{1}^{-1}, \\
w_{4} & \rightarrow w_{4} w_{2} w_{4}^{-1}, & w_{4} & \rightarrow w_{2}^{-1} .
\end{aligned}
$$

Observe that, since $\Phi_{1}$ and $\Phi_{2}$ (and hence also $\Phi_{2}^{-1}$ ) extend to $Y_{2}$, it follows from Lemma 11 that

$$
R_{j}\left(w_{1}, w_{2}, 1,1\right)=S_{j}\left(w_{1}, w_{2}, 1,1\right)=T_{j}\left(w_{1}, w_{2}, 1,1\right)=1 \quad \text { if } j=3,4 \text {. }
$$

Using this fact, and composing automorphisms to compute the action of $\Psi_{*}$, we then find that the two canonical presentations for $\pi_{1}\left(Y_{2} \cup_{\tau \Psi} Y_{2}^{\prime}\right)$ are

$$
\begin{array}{r}
\left\langle u_{1}^{\prime}, u_{2}^{\prime}: S_{3}\left(1,1, T_{1}\left(u_{1}^{\prime}, u_{2}^{\prime}, 1,1\right), T_{2}\left(u_{1}^{\prime}, u_{2}^{\prime}, 1,1\right)\right),\right. \\
\left.S_{4}\left(1,1, T_{1}\left(u_{1}^{\prime}, u_{2}^{\prime}, 1,1\right), T_{2}\left(u_{1}^{\prime}, u_{2}^{\prime}, 1,1\right)\right)\right\rangle .
\end{array}
$$




$$
\begin{aligned}
\left\langle u_{1}, u_{2}: S_{3}\left(1,1, T_{1}^{-1}\left(u_{1}, u_{2}, 1,1\right), T_{2}^{-1}\left(u_{1}, u_{2}, 1,1\right)\right),\right. & \\
& \left.S_{4}\left(1,1, T_{1}^{-1}\left(u_{1}, u_{2}, 1,1\right), T_{2}^{-1}\left(u_{1}, u_{2}, 1,1\right)\right)\right\rangle .
\end{aligned}
$$

Note also that the relators in the canonical presentation (i) for $\pi_{1}\left(Y_{2} \cup_{\tau \Phi} Y_{2}^{\prime}\right)$ are $u_{2}^{\prime 3} u_{1}^{\prime-2}$ and $u_{1}^{\prime 3} u_{2}^{\prime-4}$. Hence, composing automorphisms to obtain the action of $\Phi_{*}$, we must have

$$
\begin{aligned}
& \left\langle u_{2}^{\prime 3} u_{1}^{\prime-2}=S_{3}\left(1,1, R_{1}\left(u_{1}^{\prime}, u_{2}^{\prime}, 1,1\right), R_{2}\left(u_{1}^{\prime}, u_{2}^{\prime}, 1,1\right)\right)\right\rangle, \\
& \left\langle u_{1}^{\prime 3} u_{2}^{\prime-4}=S_{4}\left(1,1, R_{1}\left(u_{1}^{\prime}, u_{2}^{\prime}, 1,1\right), R_{2}\left(u_{1}^{\prime}, u_{2}^{\prime}, 1,1\right)\right)\right\rangle .
\end{aligned}
$$

As observed before, these are not a conjugate primitive set.

Now, since $\Phi_{1}$ and $\Phi_{2}$ (and hence also $\Phi_{2}^{-1}$ ) extend to $Y_{2}$, it follows from Lemma 13 that the set $\left\{R_{1}\left(u_{1}^{\prime}, u_{2}^{\prime}, 1,1\right), R_{2}\left(u_{1}^{\prime}, u_{2}^{\prime}, 1,1\right)\right\}$ and the set $\left\{T_{1}\left(u_{1}^{\prime}, u_{2}^{\prime}, 1,1\right), T_{2}\left(u_{1}^{\prime}, u_{2}^{\prime}, 1,1\right)\right\}$ are each bases for the free group $F_{2}$ with free basis $u_{1}^{\prime}, u_{2}^{\prime}$.

Hence the relators in the presentation (i) must be the images of $u_{2}^{\prime 3} u_{1}^{\prime-2}$ and $u_{1}^{\prime 3} u_{2}^{\prime-4}$ under a change in basis. Also $\left\{T_{1}^{-1}\left(u_{1}, u_{2}, 1,1\right), T_{2}^{-1}\left(u_{1}, u_{2}, 1,1\right)\right\}$ are a basis for $F_{2}$, hence the relators in the presentation (ii) are the image of $u_{2}^{3} u_{1}^{-2}$ and $u_{1}^{3} u_{2}^{-4}$ under a change in basis. But then Lemma 12 implies that the relators in the presentations (i) and (ii) are not conjugate primitive sets. This completes the proof of Theorem 9 .

We conclude this section with a result which relates to the special case of 2-symmetric Heegaard splittings of $S^{3}$.

THEOREM 10. Let $Y_{g} \cup_{\tau \Phi} Y_{g}^{\prime}$ be a 2-symmetric 3-manifold. Then the canonical presentations (i) and (ii) of Lemma 13 have the special property that any relators in the basis elements $u_{1}^{\prime}, \ldots, u_{g}^{\prime}$ or $u_{1}, \ldots, u_{g}$, if read backwards, remain relators.

Proof of THEOREM 10. The presentations (i) and (ii) of Lemma 10 were obtained after a particular choice of basis elements. These basis elements have the special property that each $u_{i}^{\prime}$ and each $u_{i}$ is mapped to its inverse by the involution $T_{*}$ (acting on $\pi_{1}\left(Y_{g} \cup_{\tau \Phi} Y_{g}^{\prime}\right)$ ). Since $T_{*}$ is an automorphism of $\pi_{1}\left(Y_{g} \cup_{\tau \Phi} Y_{g}^{\prime}\right)$, our theorem is established.

8. Poincare's conjecture is true for 2 -fold coverings of $S^{3}$ which are branched over closed 3-braids. Our object in this section will be to establish

THEOREM 11. If $M$ is a 2-fold covering of $S^{3}$ branched over a link defined by a closed 3-braid, then $\pi_{1} M=1$ implies $M \sim S^{3}$.

REMARK. By Lemma 1, every 3-braid knot is a knot of at most 3 bridges, and by Theorem 5 every 2 -fold covering of $S^{3}$ branched over a 3-bridge knot admits a genus 2 Heegaard splitting. Hence Theorem 11 may be regarded as a 
first step in an attempt to establish the "genus 2 Poincaré conjecture".

Proof of Theorem 11. Let $\beta$ be an element in the 3-string Artin braid group $B_{3}$, let $\widehat{\beta}$ denote the closed braid determined by $\beta$ (cf. $\S 2$ ), and let $M_{\beta}$ denote the 2 -fold covering of $S^{3}$ which is branched over $\beta$. Let $A_{\beta}(x)$ denote the Alexander polynomial of $\beta$. If $M_{\beta}$ is simply-connected, then by a result of Fox $\left[13\right.$, p. 179] it must be true that $A_{\beta}(-1)=1$. We will delineate the class of 3 braids which have this property, and show that the only possibility, if $\pi_{1} M_{\beta}=1$, is that $M_{\beta} \sim S^{3}$.

We consider two homomorphic images of the group $B_{3}$. The first, $\widetilde{B}_{3}$, is the Burau matrix group [9], [6], [21], a group of $2 \times 2$ matrices over the group ring of an infinite cyclic group, say $\langle x\rangle$. The homomorphism $\eta: B_{3} \rightarrow \widetilde{B}_{3}$ may be defined by

$$
\tilde{\sigma}_{1}=\eta\left(\sigma_{1}\right)=\left[\begin{array}{rr}
-x & 0 \\
x & 1
\end{array}\right], \quad \tilde{\sigma}_{2}=\eta\left(\sigma_{2}\right)=\left[\begin{array}{rr}
1 & 1 \\
0 & -x
\end{array}\right]
$$

where $\sigma_{1}$ and $\sigma_{2}$ are standard generators of $B_{3}$ (cf. $\S 2$ ). It was established by Burau [9] that these matrices are closely related to Alexander matrices of closed braids; explicitly, if $\widetilde{\beta}=\eta(\beta)$, then

$$
A_{\beta}(x)=\operatorname{det}|\tilde{\beta}-I| /(1+x+x)^{2}, .
$$

where $I$ is the $2 \times 2$ identity matrix.

Now, we are interested in $A_{\beta}(-1)$, hence we may define a second homomorphism $\xi: \widetilde{B}_{3} \rightarrow \hat{B}_{3}$, defining $\hat{B}_{3}$ to be the matrix group obtained by replacing the indeterminate " $x$ " by " -1 ". The group $\hat{B}_{3}$ is easily recognized to be the group of $2 \times 2$ matrices with integral entries and determinant +1 , for on setting $x=-1$ in the matrices $\tilde{\sigma}_{1}$ and $\tilde{\sigma}_{2}$ we obtain

$$
\hat{\sigma}_{1}=\xi\left(\tilde{\sigma}_{1}\right)=\left[\begin{array}{rr}
1 & 0 \\
-1 & 1
\end{array}\right], \quad \hat{\sigma}_{2}=\xi\left(\tilde{\sigma}_{2}\right)=\left[\begin{array}{ll}
1 & 1 \\
0 & 1
\end{array}\right],
$$

which are known to generate the latter group [10, p. 85]. Defining relations in $\hat{B}_{3}$ are $[10]$ :

$$
\begin{gathered}
\sigma_{1} \sigma_{2} \sigma_{1}=\sigma_{2} \sigma_{1} \sigma_{2}, \\
\left(\sigma_{1} \sigma_{2}\right)^{6}=1 .
\end{gathered}
$$

Since relation (36) lifts to the single defining relation in $B_{3}$, and since $\left(\sigma_{1} \sigma_{2}\right)^{6}$ is known to belong to the center of $B_{3}$, it then follows that

$$
\operatorname{ker}(\xi \eta)=\text { cyclic subgroup of } B_{3} \text { generated by }\left(\sigma_{1} \sigma_{2}\right)^{6} \text {. }
$$

To delineate the class of 3-braids which have the property that $A_{\beta}(-1)=1$, suppose that 


$$
\hat{\beta}=\xi \eta(\beta)=\left[\begin{array}{ll}
a & b \\
c & d
\end{array}\right], \quad a d-b c=1, a, b, c, d \text { integers. }
$$

Equation (34) then implies that

$$
A_{\beta}(-1)=\left|\begin{array}{cc}
a-1 & b \\
c & d-1
\end{array}\right|=2-a-d .
$$

Since $A_{\beta}(x)$ is only defined up to multiplication by an arbitrary power of $x$, we must consider two cases, namely $2-a-d= \pm 1$, or $a+d=1$ or 3 . It is proved in [21, Lemma 2.3] that if trace $\hat{\beta}=1$ or 3 , then $\hat{\beta}$ is conjugate to one of the elements $\sigma_{1} \sigma_{2}, \sigma_{2}^{-1} \sigma_{1}^{-1}$, or $\sigma_{2} \sigma_{1}^{-1}$. Using equation (38) above, we then find that $A_{\beta}(-1)=1$ if and only if $\beta$ is conjugate in $B_{3}$ to one of the elements $\left(\sigma_{1} \sigma_{2}\right)^{6 m+1}$, $\left(\sigma_{1} \sigma_{2}\right)^{6 m-1}, \sigma_{2} \sigma_{1}^{-1}\left(\sigma_{1} \sigma_{2}\right)^{6 m}, m=0, \pm 1, \pm 2, \ldots$ Since conjugate braids determine equivalent closed braids (in the sense of link equivalence), we have thus proved that $A_{\beta}(-1)=1$ implies

$$
\begin{aligned}
\beta=\beta_{m}^{(1)}=\left(\sigma_{1} \sigma_{2}\right)^{6 m \pm 1} \text { or } \beta_{m}^{(2)}=\left(\sigma_{2} \sigma_{1}^{-1}\right)\left(\sigma_{1} \sigma_{2}\right)^{6 m}, & \\
& m=0, \pm 1, \pm 2, \ldots
\end{aligned}
$$

To conclude the proof, we examine the class of links determined by the closed braids enumerated above. A simple picture shows that if $m \neq 0$, the knots defined by the braids $\beta_{m}^{(1)}$ are torus knot types, hence their groups have nontrivial centers. By a recent result of Gordon and Heil [15], if $M$ is a simply-connected 3-manifold which is represented as a covering of $S^{3}$ of finite degree branched over a knot which has a group with a nontrivial center, then in fact $M$ must be $S^{3}$. On the other hand, suppose $\beta=\beta_{m}^{(2)}, m \neq 0$. Then, an argument given by J. Montesinos in [25] shows that the 2-fold covering of $S^{3}$ branched over the knot determined by $\beta_{m}^{(2)}$ is homeomorphic to the three-manifold obtained by cutting out a "twist knot" from $S^{3}$ and sewing it back differently. By results of Bing and Martin [35], a simply-connected 3-manifold cannot be obtained from $S^{3}$ by surgery on a nontrivial twist knot. Therefore the only case where we might find a homotopy sphere which is not a 3 -sphere is if $\beta=\beta_{n}^{1}$ or $\beta_{n}^{2}$, but in this case $\beta=$ is easily seen to define the trivial knot type, so that in fact $M$ is $S^{3}$. This concludes the proof of Theorem 10.

\section{REFERENCES}

1. J. W. Alexander, A note on Riemann space, Bull. Amer. Math. Soc. 26 (1919), 370-372.

2. - A lemma on systems of knotted curves, Proc. Nat. Acad. Sci. U.S.A. 9 (1923), 93-95.

3. E. Artin, Theorie der Zöpfe, Abh. Math. Sem. Univ. Hamburg 4 (1925), 47-72.

4. - Theory of braids, Ann. of Math. (2) 48 (1947), 101-126. MR 8, 367.

5. J. S. Birman, Plat presentations for link groups, Comm. Pure Appl. Math. 26

(1973), 673-678. 
6. J. S. Birman, Braids, links and mapping class groups, Ann. of Math. Studies, no. 82, Princeton Univ. Press, Princeton, N. J., 1975.

7. J. S. Birman and H. M. Hilden, On the mapping class groups of closed surface as covering spaces, Advances in the Theory of Riemann Surfaces (Proc. Conf., Stony Brook, N. Y., 1969), Ann. of Math. Studies, no. 66, Princeton Univ. Press, Princeton, N. J., 1971, pp. 81-115. MR 45 \#1169.

8. The homeomorphism problem for $S^{3}$, Bull. Amer. Math. Soc. 79 (1973), 1006-1010. MR 47 \#7726.

9. W. Burau, Über Zopfgruppen und gleichsinnig verdrillte Verkettungen, Abh. Math. Sem. Hansischen Univ. 11 (1936), 171-178.

10. H. S. M. Coxeter and W. O. J. Moser, Generators and relations for discrete groups, 2nd ed., Ergebnisse der Mathematik und ihrer Grenzgebiete, N. F., Band 14, Springer-Verlag, Berlin and New York, 1965. MR 30 \#4818.

11. M. Dehn, Die Gruppe der Abbildungsklassen, Acta Math. 69 (1938), 135-206.

12. R. H. Fox, On the total curvature of some tame knots, Ann. of Math. (2) 52 (1950), 258-260. MR 12, 273.

13. Knots and periodic transformations, Topology of 3-Manifolds and Related Topics (Proc. Univ. of Georgia Inst., 1961), Prentice-Hall, Englewood Cliffs, N. J., 1962, pp. 177-182. MR 25 \#3524.

14. R. H. Fox and L. Neuwirth, The braid groups, Math. Scand. 10 (1962), 119-

126. MR 27 \#742.

15. C. McA. Gordon and W. Heil, Simply-connected branched coverings of $S^{3}$, Proc.

Amer. Math. Soc. 35 (1972), 287-288. MR 45 \#5989. \#4519a.

16. W. Haken, Theorie der Normalflichen, Acta Math. 105 (1961), 245-375. MR 25

17. W. B. R. Lickorish, A representation or orientable, combinatorial 3-manifolds, Ann. of Math. (2) 76 (1962), 531-540. MR 27 \#1929.

18. - A finite set of generators for the homeotopy group of a 2-manifold, Proc. Cambridge Philos. Soc. 60 (1964), 769-778; Corrigendum, ibid. 62 (1966), 679-681.

MR 30 \#1500; 34 \#799.

19. W. Magnus, Über Automorphismen von Fundamental-Gruppen berandeter Fld̈chen, Math. Ann. 109 (1934), 617-646.

20. W. Magnus, A. Karrass and D. Solitar, Combinatorial group theory: Presentations of groups in terms of generators and relations, Pure and Appl. Math., vol. 13, Interscience, New York, 1966. MR 34 \#7617.

21. W. Magnus and A. Pelluso, On knot groups, Comm. Pure Appl. Math. 20 (1967), 749-770. MR 36 \#5930.

22. W. S. Massey, Algebraic topology: An introduction, Harcourt, Brace \& World, New York, 1967. MR 35 \#2271.

23. J. W. Milnor, On the total curvature of knots, Ann. of Math. (2) 52 (1950), 248-257. MR 12, 273.

24. José $M$. Montesinos, 3-variétés que ne sont pas revêtements cycliques remifiés sur $S^{3}$, Proc. Amer. Math. Soc. 47 (1975), 495-500.

25. - Surgery of links for double branched covers of $S^{3}$, Ann. of Math.

Studies, no. 184, Princeton Univ. Press, Princeton, N. J., 1975.

26. K. Murasugi, On closed 3-braids, Mem. Amer. Math. Soc., no. 151, 1974.

27. C. D. Papakyriakopoulos, Dehn's Lemma and the asphericity of knots, Ann. of

Math. (2) 96 (1957), 1-26.

28. - Some problems on 3-dimensional manifolds, Bull. Amer. Math. Soc. 64 (1958), 317-335. MR 21 \#1600.

29. K. Reidemeister, Knoten und Geflechte, Nachr. Akad. Wiss. Göttingen Math.-Phys. K1. II 1960, 105-115. MR 22 \#1913.

30. H. Schubert, Knoten mit zwei Brücken, Math. Z. 65 (1956), 133-170. MR 18, 498.

31. Bestimmung der Primfaktorzerlegung von Verkettung, Math. Z. 76 (1961), 116-148. MR 25 \#4519b. 
32. O. Ja. Viro, Linkings, two-sheeted branched coverings, and braids, Mat. Sb. 87 (129) (1972), 216-228 = Math. USSR Sb. 16 (1972), 223-236. MR 45 \#7701.

33. F. Waldhausen, Heegaard-Zerlegungen der 3-Sphdre, Topology 7 (1968), 195-203. MR 37 \#3576.

34. —, Über Involutioen der 3-Sphare, Topology 8 (1969), 81-91. MR 38 \#5209.

35. R. H. Bing and J. M. Martin, Cubes with knotted holes, Trans. Amer. Math. Soc. 155 (1971), 217-231. MR 43 \#4018a.

DEPARTMENT OF MATHEMATICS, COLUMBIA UNIVERSITY, NEW YORK, NEW YORK 10027

DEPARTMENT OF MATHEMATICS, UNIVERSITY OF HAWAII, HONOLULU, HAWAII 96822 